\title{
The role of hydrogen bonds in formation of co-amorphous valsartan/nicotinamide compositions of high solubility and durability with anti-hypertension and anti-COVID-19 potential
}

Marika Turek a*, Ewa Różycka-Sokołowska a*, Marek Koprowski b, Bernard Marciniak a, Piotr Bałczewski a,b*

a Institute of Chemistry, Faculty of Science and Technology, Jan Długosz University in Częstochowa, Armii Krajowej 13/15, Częstochowa, 42-201, Poland;

b Division of Organic Chemistry, Centre of Molecular and Macromolecular Studies, Polish Academy of Sciences, Sienkiewicza 112, Łódź, 90-363, Poland.

* Piotr Bałczewski, e-mail: pbalczew@cbmm.lodz.pl

* Marika Turek, e-mail: marika.turek@ajd.czest.pl;

* Ewa Różycka-Sokołowska, e-mail: e.sokolowska@ajd.czest.pl

\section{SUPPLEMENTARY MATERIAL}

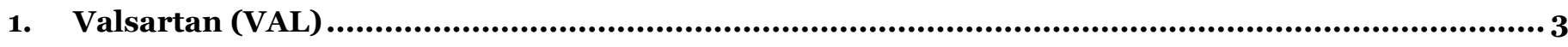

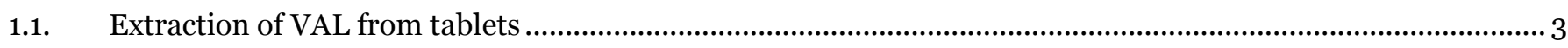

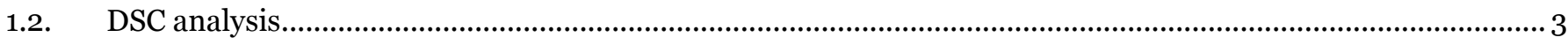

Fig. S1. DSC curve of VAL extracted from tablets. ......................................................................................... 3

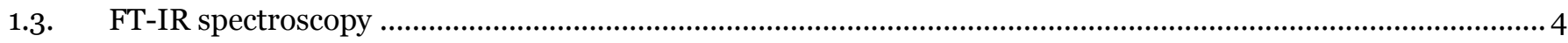

Fig. S2. The IR spectrum of VAL extracted from tablets. ...........................................................................

Table S1. FT-IR characteristic vibrations of isolated VAL compared with published data 4,5 .................................. 4

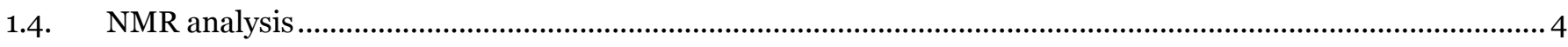

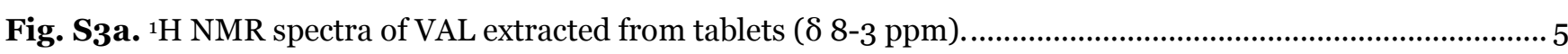

Fig. S3b. ${ }^{1} \mathrm{H}$ NMR spectra of VAL extracted from tablets $(\delta$ 3-0 ppm). .................................................................. 5

Fig. S4. ${ }^{13} \mathrm{C}$ NMR spectra of VAL extracted from tablets. .......................................................................................... 6

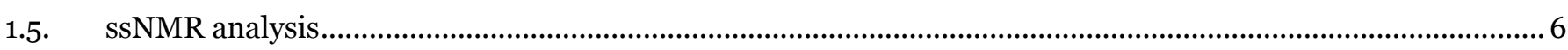

Fig. S5. ${ }^{13} \mathrm{C}$ ssNMR spectra of VAL extracted from tablets and purchased (referenced). Asterisks denote spinning sidebands.

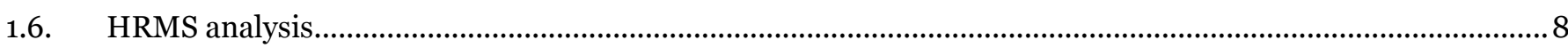

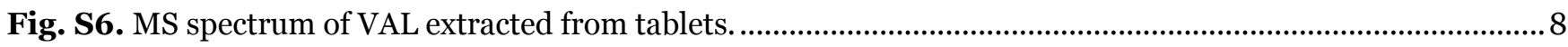

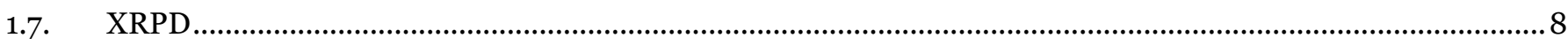

Fig. S7. XRPD pattern of (a) VAL used in this study; (b) VAL purely amorphous; (c) VAL polymorphic form VII.9

2. Nicotinamide (NIC)

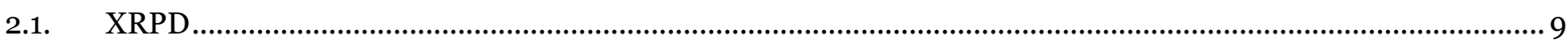

Fig. S8. XRPD pattern of NIC used in this study along with designation of major reflexes. .................................. 9

3. Solid dispersions (VAL/NIC) ............................................................................................. 10

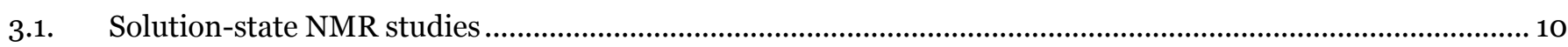

Fig. S9. ${ }^{1} \mathrm{H}$ NMR spectra for (a) VAL/NIC slurry, (b) VAL/NIC LAG $30 \mathrm{~min}$, (c) VAL/NIC LAG 60 min, (d) VAL/NIC ball mill along with calculations regarding the content of EtOH..............................................................11

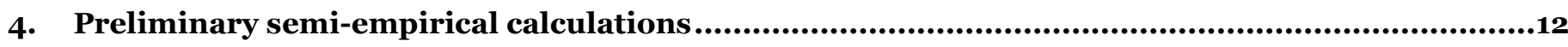

Fig. S10. Chemical structures of 5 possible VAL/NIC heterodimers optimized by using AM1 method along with calculated total energy (Hartree). 
Table S2. Atom coordinates $(\AA)$, total energy (Hartree) and the number of imaginary vibrational frequencies for the geometry of VAL optimized at the B3LYP/6-311++(d,p) level in the gas phase using Gaussian o9.

Table S3. Atom coordinates $(\AA)$, total energy (Hartree) and the number of imaginary vibrational frequencies for the geometry of NIC optimized at the B3LYP/6-311++(d,p) level in the gas phase using Gaussian 09.

Table S4a. Atom coordinates $(\AA)$, total energy (Hartree) and the number of imaginary vibrational frequencies for the geometry of VAL/NIC1 optimized at the B3LYP/6-311++(d,p) level in the gas phase using Gaussian o9.

Table S4b. Atom coordinates $(\AA)$, total energy (Hartree) and the number of imaginary vibrational frequencies for the geometry of VAL/NIC1 optimized at the B3LYP/6-311++(d,p) level in the ethanol using Gaussian o9. 16

Table S5a. Atom coordinates $(\AA)$, total energy (Hartree) and the number of imaginary vibrational frequencies for the geometry of VAL/NIC2 optimized at the B3LYP/6-311++(d,p) level in the gas phase using Gaussian o9.

Table S5b. Atom coordinates $(\AA)$, total energy (Hartree) and the number of imaginary vibrational frequencies for the geometry of VAL/NIC2 optimized at the B3LYP/6-311++(d,p) level in the ethanol using Gaussian o9. 19

5.2. Vibrational spectra

Table S6. Comparison of experimental vibrations of VAL and NIC with calculated frequencies along with potential energy distribution (PED, \%).

Fig. S11. Comparison of the experimental FT-IR spectrum of VAL (a) and NIC (b) with the theoretical frequencies.

Table S7. Comparison of experimental vibrations of VAL/NIC slurry with calculated frequencies. 25

Table S8. Comparison of experimental vibrations of VAL/NIC LAG 60 min. with calculated frequencies. .... 25

Table S9. Comparison of experimental vibrations of VAL/NIC ball mill with calculated frequencies..............25

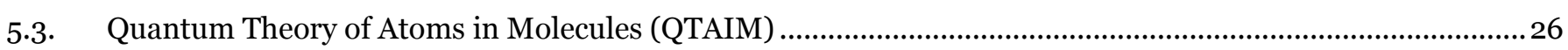

Table S10. Topological parameters corresponding to H-bonds involved in intermolecular interactions. ............26

Table S11. Geometrical parameters corresponding to H-bonds involved in intermolecular interactions along with molecular graphs with the BCPs $(3,-1)$ as orange dots.

Fig. S12a. 3D-NCI plot with color-filled reduced density gradient (RDG) isosurfaces depicting non-covalent interactions in VAL/NIC1 and VAL/NIC2 along with 2D-NCI scatter plots (calculated in gas phase)...................28

Fig. S12b. 3D-NCI plot with color-filled reduced density gradient (RDG) isosurfaces depicting non-covalent interactions in VAL/NIC1 and VAL/NIC2 along with 2D-NCI scatter plots (calculated in ethanol).

Table S12. Topological parameters corresponding to H-bonds involved in intramolecular interactions in homodimers.

Table S13. Geometrical parameters determined from X-ray structures, corresponding to H-bonds involved in intramolecular interactions in homodimers.

Fig. S13. 3D-NCI plot with color-filled reduced density gradient (RDG) isosurfaces depicting non-covalent interactions in homodimers along with $2 \mathrm{D}-\mathrm{NCI}$ scatter plots. 


\section{Valsartan (VAL)}

\subsection{Extraction of VAL from tablets}

Valsartan (VAL) was extracted from tablets of Vanatex (Polpharma SA) and Axudan (Sandoz GmbH). The tablets without envelopes were crushed by using a mortar and pestle, and VAL was extracted with acetonitrile. It was purified by a slurry crystallization from the $n$-heptane and diethyl ether $(\mathrm{v} / \mathrm{v}, 1: 1)$ mixture $\left(30^{\circ} \mathrm{C}, 72 \mathrm{~h}\right) .{ }^{1} \mathrm{Then}$, the mixture was cooled, filtered and the residue was dried under vacuum for $24 \mathrm{~h}$ to give pure VAL in $64 \%$ yield (m.p. $114-118^{\circ} \mathrm{C}$ ), which is consistent with literature data. ${ }^{2}$

The ${ }^{1} \mathrm{H}$ NMR and ${ }^{13} \mathrm{C}$ NMR spectra were measured on a Bruker AV III 500 spectrometer in MeOD with chemical shifts $(\delta)$ given in ppm relative to TMS as an internal standard. The ${ }^{13} \mathrm{C}$ cross-polarization magic angle spinning ${ }^{13} \mathrm{C}$ CPMAS), ssNMR experiments were performed on a Bruker Avance III 600 spectrometer with resonance frequencies of $150.918 \mathrm{MHz}$. The spectra were collected with a spinning rate of $12 \mathrm{kHz}$ and the relaxation delays (RDs) during the acquisitions of $5 \mathrm{~s}$ and $300 \mathrm{~s}$. The Fourier transformation mid infrared (MIR) spectra of VAL in the region 4000-400 $\mathrm{cm}^{-1}$ were measured at $2 \mathrm{~cm}^{-1}$ resolution with co-addition of 32 scans on a Nicolet-Nexus spectrometer using the $\mathrm{KBr}$ pellet technique. The differential scanning calorimetry (DSC) measurements were performed using a Netzsch STA $409 \mathrm{C} / \mathrm{CD}$ instrument. The samples were placed in sealed non-hermetic aluminum pans and scanned at a heating rate $10.0 \mathrm{~K} / \mathrm{min}$ under an argon atmosphere. High-resolution mass spectrometry (HRMS) measurements were performed using Synapt G2-Si mass spectrometer (Waters) equipped with an ESI source and quadrupole-Time-of-Flight mass analyser. The mass spectrometer was operated in the positive ion detection mode. The measurement was performed with capillary voltage set to $2.7 \mathrm{kV}$ and sampling cone to $20 \mathrm{~V}$. The source temperature was $110^{\circ} \mathrm{C}$. The results of the measurements were processed using the MassLynx 4.1 software (Waters) incorporated with the instrument. X-ray powder diffraction (XRPD) data were collected at room temperature on an Xcalibur ${ }^{\mathrm{TM}} 3 \mathrm{CCD}$ diffractometer using $\mathrm{CuK} \alpha$ radiation. XRPD experiments were performed using transmission geometry at room temperature with powdered samples sealed in capillary tubes and rotated about Phi over $360^{\circ}$ at $0.5^{\circ} / \mathrm{S}$. CCD image data were processed by CrysAlisPRO software. The obtained data were analyzed by using the Fullprof software.

\subsection{DSC analysis}

Nalluri et al. reported that recrystallization of valsartan from methanol, ethanol, isopropanol, and acetonitrile yields several endothermic (broad) peaks according to their DSC thermograms. ${ }^{3}$ The DSC curve of the obtained VAL shows the tallest endothermic peak $\left(\mathrm{T}_{\mathrm{m}}\right)$ at $117.9^{\circ} \mathrm{C}$ with enthalpy $29.25 \mathrm{~J} / \mathrm{g}$ related to the melting point and glass transition $\left(\mathrm{T}_{\mathrm{g}}\right)$ event at $69.8^{\circ} \mathrm{C}$ (Fig. S1), what is consistent with literature data, where the reported glass transition events of valsartan were at 67,69 and $78{ }^{\circ} \mathrm{C} .4$ Decomposition of valsartan starts around $160^{\circ} \mathrm{C}$, which is consistent with the experimental DSC curve.

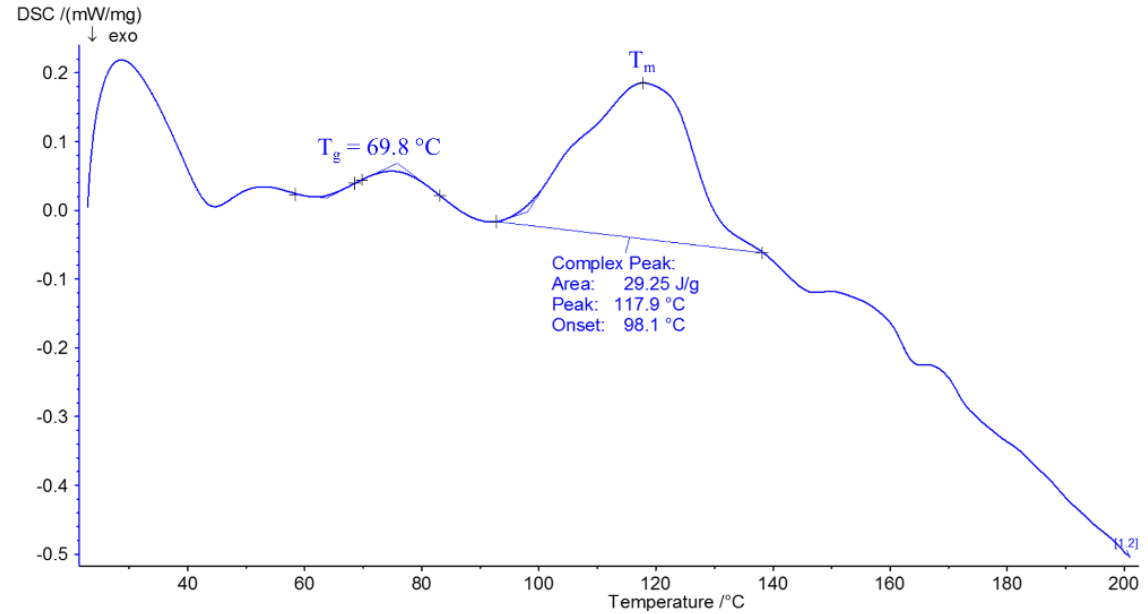

Fig. S1. DSC curve of VAL extracted from tablets.

\footnotetext{
1 J.R. Wang, X. Wang, L. Lu, X. Mei, Highly crystalline forms of valsartan with superior physicochemical stability. Cryst. Growth Des., 2013, 13 (7), $3261-3269$.

${ }^{2}$ Merck \& Co., Inc., The Merck Index, Merck \& Co., Inc., Whitehouse Station, NJ, USA, 2008.

${ }^{3}$ B.N. Nalluri, R.M. Krishna, T.P. Rao, P.A. Crooks, Effect of recrystallization on the pharmaceutical properties of valsartan for improved therapeutic efficacy, J. Appl. Pharm. Sci., 2012, 2 (10), 126-132.

4 J. J. M. Ramos, H. P. Diogo, Thermal behavior and molecular mobility in the glassy state of three anti-hypertensive pharmaceutical ingredients, RSC Adv., 2017, 7 , 10831-10840.
} 


\subsection{FT-IR spectroscopy}

The FT-IR spectrum of VAL in the KBr pellet is shown in Fig. $\mathbf{2} 2$ with main peaks and their assignments listed in

Table S1. The IR characteristic vibrations of VAL are consistent with the literature data.5,6

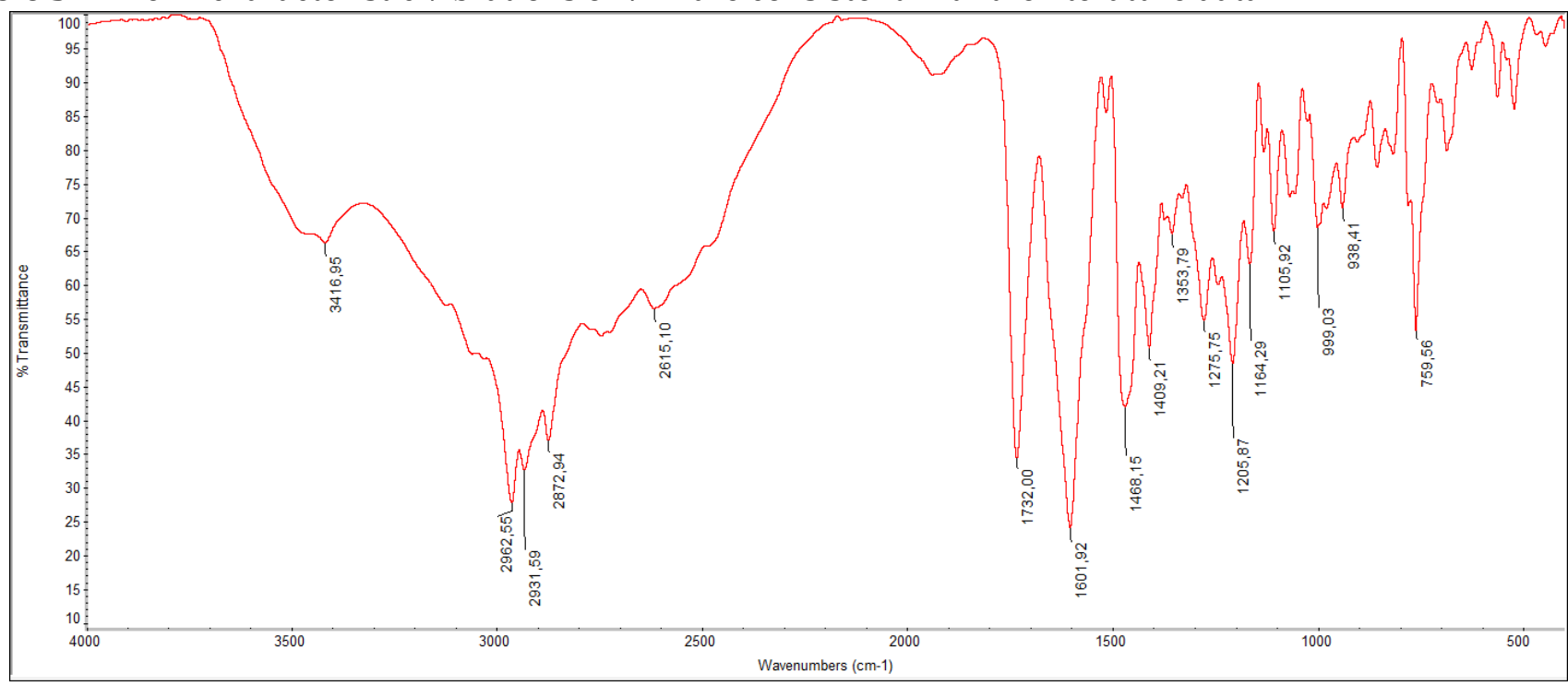

Fig. S2. The IR spectrum of VAL extracted from tablets.

Table S1. FT-IR characteristic vibrations of isolated VAL compared with the published data ${ }^{4,5}$

\begin{tabular}{|c|c|c|}
\hline \multirow{2}{*}{ Assignment } & \multicolumn{2}{|c|}{ Wavenumber $\left[\mathrm{cm}^{-1}\right]$} \\
\hline & Experimental & Literature \\
\hline$v(\mathrm{OH})$ & 3121 & $3121^{4}$ \\
\hline$v(\mathrm{NH})$ & 3416 & $3435^{4}$ \\
\hline$v(C=0)^{\text {acid }}$ & 1732 & $1733^{4}, 1602^{5}$ \\
\hline$v(\mathrm{C}=0)^{\text {amide }}$ & 1602 & $1603^{4}, 1602^{5}$ \\
\hline$v(C-0)$ & 1396 & $1391^{4}$ \\
\hline
\end{tabular}

$v$ - stretching

\subsection{NMR analysis}

Two cis/trans rotamers of valsartan, major and minor ones have been reported by Li et al. who suggested that these species existed as the result of rotation around the amide $\mathrm{C}(\mathrm{O})-\mathrm{N}$ bond.7 Further studies were conducted by Chashmniam and Tafazzoli, who identified four different rotamers. ${ }^{8}$ Consequently, ${ }^{1} \mathrm{H}$ and ${ }^{13} \mathrm{C}$ NMR spectra of valsartan indicated two sets of data which are presented in Fig. S3 and S4. Calculated major/minor rotamers ratio is $2: 1(\mathrm{~m} / \mathrm{M}=0.50$ ratio $)$ and is consistent with the literature data, where $\mathrm{m} / \mathrm{M}=0.48$ ratio was reported in MeOD.7

${ }^{5}$ K. R. Rajeswari, K. Abbulu, M. Sudhakar, R. Karki, B. Rajkumar, Development and characterization of valsartan loaded hydrogel beads, Pharm. Lett., 2012, 4 (4), 10441053

${ }^{6}$ A. Sampath, A.R. Reddy, B. Yakambaran, A. Thirupathi, M. Prabhakar, P.P. Reddy, V.P. Reddy, Identification and characterization of potential impurities of valsartan AT1 receptor antagonist, J. Pharm. Biomed. Anal., 2009, 50, 405-412.

${ }^{7}$ F. Li, H. Zhang, L. Jiang, W. Zhang, J. Nie, Y. Feng, M. Yang, M. Liu, Dynamic NMR study and theoretical calculations on the conformational exchange of valsartan and related compounds, Magn. Reson. Chem., 2007, 45, 929-936.

${ }^{8} \mathrm{~S}$. Chashmniam, M. Tafazzoli, NMR investigation and theoretical calculations of the solvent effect on the conformation of valsartan, J. Mol. Struct., 2017, 1148, 73-80. 


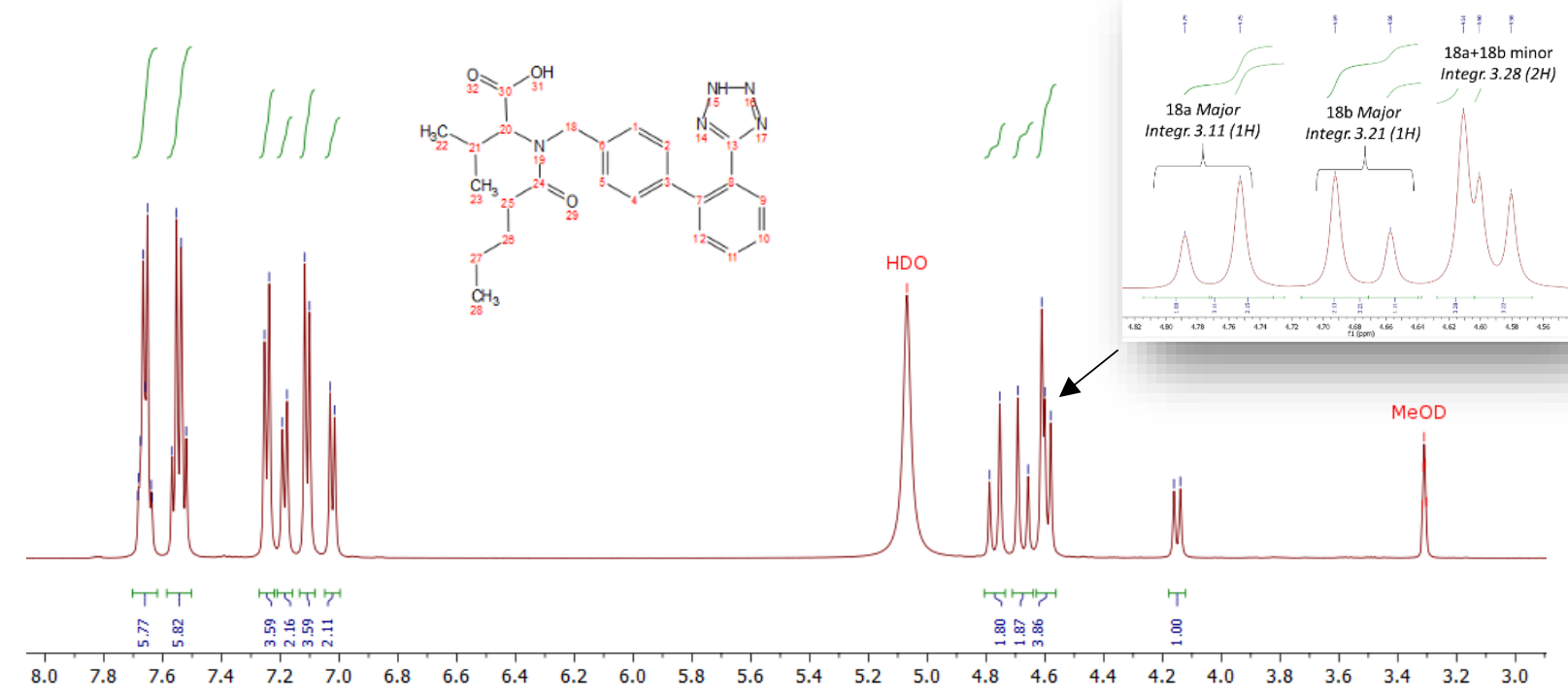

Fig. S3a. ${ }^{1} \mathrm{H}$ NMR (MeOD) spectra of VAL extracted from tablets $(\delta 8-3 \mathrm{ppm})$.

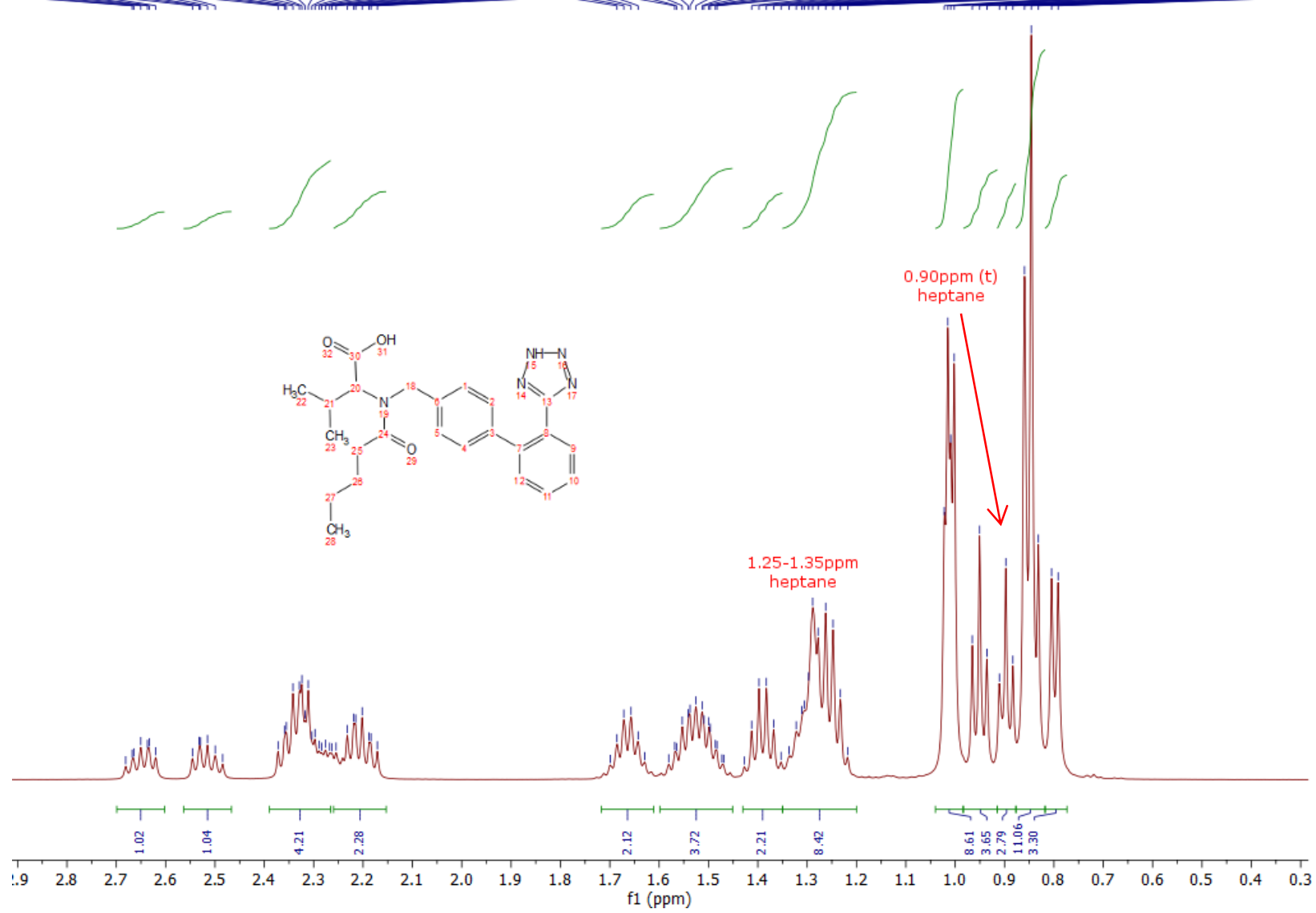

Fig. S3b. ${ }^{1} \mathrm{H}$ NMR (MeOD) spectra of VAL extracted from tablets ( $\left.\delta 3-0 \mathrm{ppm}\right)$.

* the residual heptane comes from the crystallization process

${ }^{1} \mathrm{H}$ NMR (major conformer) (500 MHz, MeOD): $\delta 7.54(\mathrm{q}, J=8.1 \mathrm{~Hz}, 4 \mathrm{H}), 7.25(\mathrm{~d}, J=7.9 \mathrm{~Hz}, 1 \mathrm{H}), 7.24(\mathrm{~s}, 1 \mathrm{H}), 7.12$ (s, 1H), $7.11(\mathrm{~d}, J=7.9 \mathrm{~Hz}, 1 \mathrm{H}), 4.77(\mathrm{~d}, J=17.5 \mathrm{~Hz}, 1 \mathrm{H}), 4.67(\mathrm{~d}, J=17.5 \mathrm{~Hz}, 1 \mathrm{H}), 4.59$ (d, $J=10.0 \mathrm{~Hz}, 1 \mathrm{H}), 2.25-$ 
$2.37(\mathrm{~m}, 1 \mathrm{H}), 2.33(\mathrm{~s}, 1 \mathrm{H}), 2.17-2.23(\mathrm{~m}, 1 \mathrm{H}), 1.37-1.43(\mathrm{~m}, 2 \mathrm{H}), 1.22-1.34(\mathrm{~m}, 2 \mathrm{H}), 1.01(\mathrm{~d}, J=6.5,3.5 \mathrm{~Hz}, 3 \mathrm{H})$, $0.83-0.86(\mathrm{~m}, 3 \mathrm{H}), 0.80(\mathrm{~d}, J=6.7 \mathrm{~Hz}, 3 \mathrm{H})$.

${ }^{1} \mathrm{H}$ NMR (minor conformer) (500 MHz, MeOD): $\delta$ 7.65-7.68 (m, 4H), $7.19(\mathrm{~d}, J=7.9 \mathrm{~Hz}, 1 \mathrm{H}), 7.18(\mathrm{~s}, 1 \mathrm{H}), 7.03(\mathrm{~s}$, $1 \mathrm{H}), 7.02(\mathrm{~d}, J=7.9 \mathrm{~Hz}, 1 \mathrm{H}), 4.61(\mathrm{~s}, 2 \mathrm{H}), 4.15(\mathrm{~d}, J=10.7 \mathrm{~Hz}, 1 \mathrm{H}), 2.62-2.68(\mathrm{~m}, 1 \mathrm{H}), 2.48-2.55(\mathrm{~m}, 1 \mathrm{H}), 2.22(\mathrm{~s}$, $1 \mathrm{H}), 1.63-1.70(\mathrm{~m}, 2 \mathrm{H}), 1.47-1.58(\mathrm{~m}, 2 \mathrm{H}), 1.02(\mathrm{~d}, J=3.5 \mathrm{~Hz}, 3 \mathrm{H}), 0.95(\mathrm{t}, J=7.4 \mathrm{~Hz}, 3 \mathrm{H}), 0.83-0.86(\mathrm{~m}, 3 \mathrm{H})$.

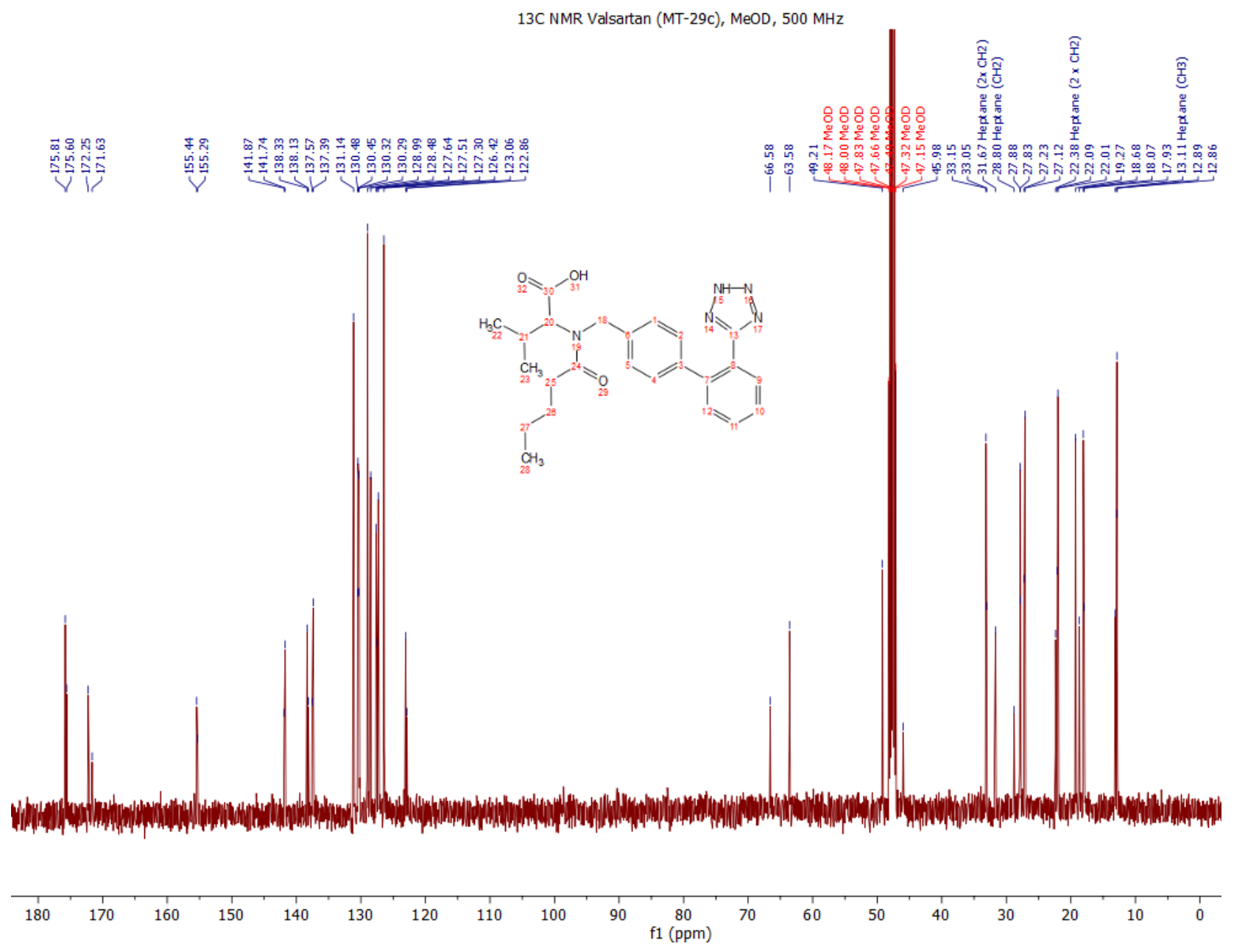

Fig. S4. ${ }^{13} \mathrm{C}$ NMR (MeOD) spectra of VAL extracted from tablets.

$*$ the residual heptane comes from the crystallization process

${ }^{13} \mathrm{C}$ NMR (major conformer) (126 MHz, MeOD) $\delta 175.81$ (C-24), 172.25 (C-30), 155.44 (C-13), 141.87 (C-7), 138.33 (C-3), 137.57 (C-6), 131.14 (C-9), 130.45 (C-11), 130.32 (C-12) 128.99 (C-4, C-8), 128.48 (C-2), 126.42 (C-1), 127.51 (C-5), 123.06 (C-10), 63.58 (C-20), 49.21 (C-18), 33.15 (C-25), 27.88 (C-21), 27.23 (C-26), 22.09 (C-27), 19.27 (C-22), 18.07 (C-23), 12.89 (C-28).

${ }^{13} \mathrm{C}$ NMR (minor conformer) (126 MHz, MeOD) $\delta 175.60$ (C-24), 171.63 (C-30), 155.29 (C-13), 141.74 (C-7), 138.13 (C-3), 137.39 (C-6), 131.14 (C-9), 130.48 (C-11), 130.29 (C-12), 128.99 (C-4), 127.64 (C-2), 127.30 (C-5), 126.42 (C-1, C-8), 122.86 (C-10), 66.58 (C-20), 45.98 (C-18), 33.05 (C-25), 27.83 (C-21), 27.12 (C-26), 22.01 (C27), 18.68 (C-22), 17.93 (C-23), 12.86 (C-28).

\section{5. ssNMR analysis}

The structure of the solid form of VAL was also confirmed by utilizing solid-state NMR (ssNMR). Spectra were recorded with the spinning rate of $12 \mathrm{kHz}$ and relaxation delays (RDs) equal $5 \mathrm{~s}$ and $300 \mathrm{~s}$. The ${ }^{13} \mathrm{C}$ ssNMR spectra of VAL extracted from tablets were compared with the same spectra of the VAL sample purchased from SigmaAldrich. The spectra recorded with RDs of $5 \mathrm{~s}$ and $300 \mathrm{~s}$ were identical; both in case of VAL extracted from tablets and VAL purchased (Fig. S5). 

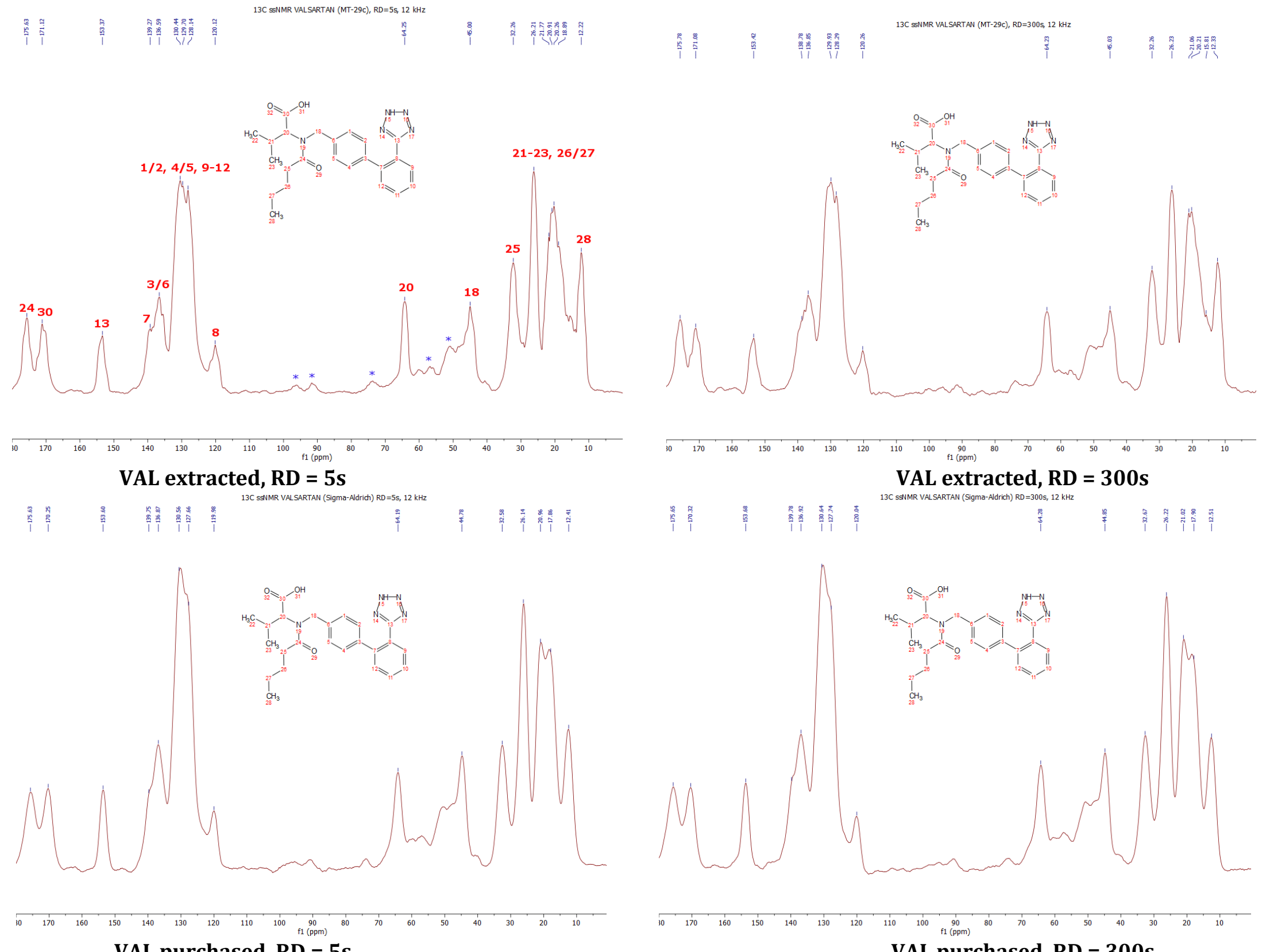

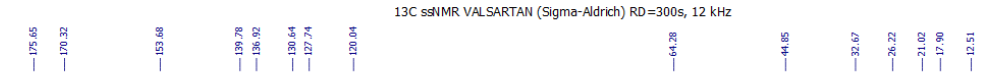

VAL purchased, $\mathrm{RD}=\mathbf{5 s}$

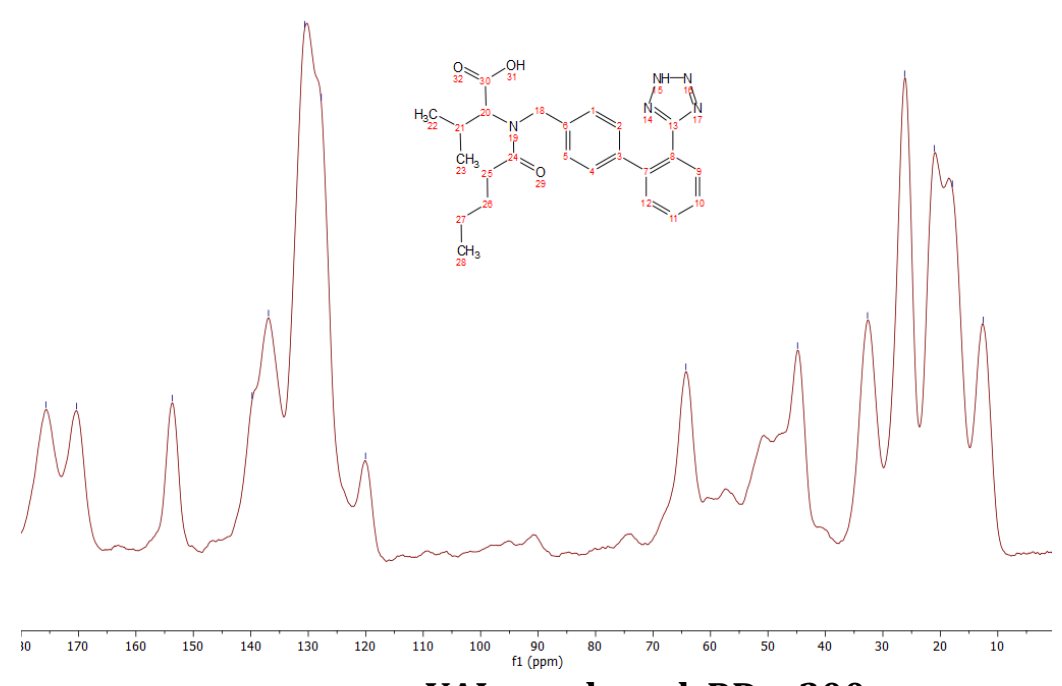

VAL purchased, $\mathrm{RD}=\mathbf{3 0 0 \mathrm { s }}$

Fig. S5. ${ }^{13} \mathrm{C}$ ssNMR spectra of VAL extracted from tablets and purchased (Sigma-Aldrich, as a reference).Asterisks denote spinning sidebands. 


\subsection{HRMS analysis}

The ESI-MS spectrum of VAL used in this study is shown in Fig. S6. The MS data are similar to that described in the literature. ${ }^{9}$ The important fragments $\left(\mathrm{m} / \mathrm{z}\right.$ ) are 436 (valsartan (acid), $[\mathrm{M}+\mathrm{H}]^{+}$) and 458 (valsartan sodium salt, $[\mathrm{M}+\mathrm{Na}]^{+}$).

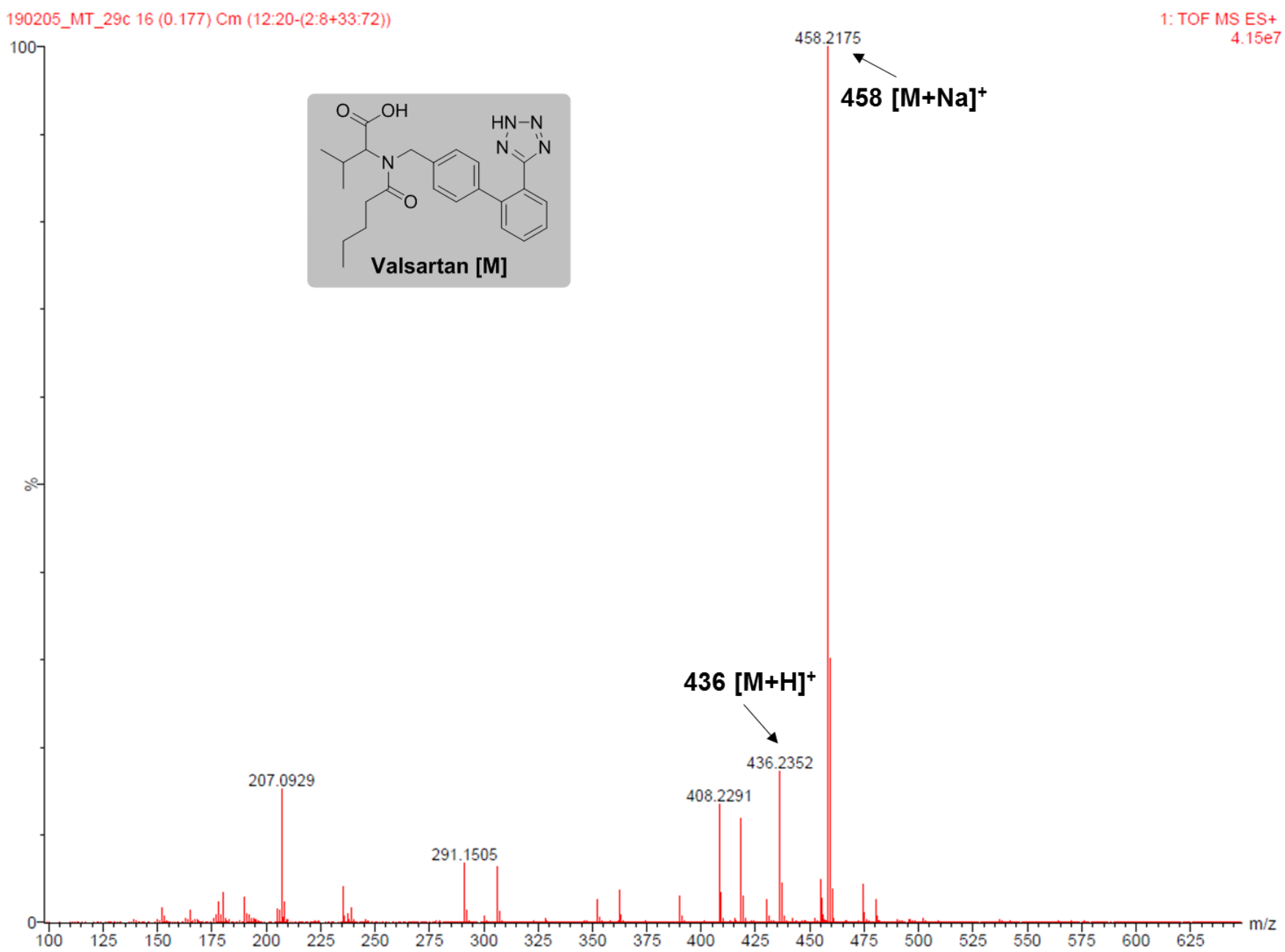

Fig. S6. MS spectrum of VAL extracted from tablets.

MS - ES (+): $\mathrm{m} / \mathrm{z}(\%)=436\left[(\mathrm{M}+\mathrm{H})^{+}, 20\right] ; 458\left[(\mathrm{M}+\mathrm{Na})^{+}, 100\right]$

HRMS (TOF MS ES+) (m/z): calcd for $\mathrm{C}_{24} \mathrm{H}_{30} \mathrm{~N}_{5} \mathrm{O}_{3}[\mathrm{M}+\mathrm{H}]+: 436.2349$; found: 436.2352 .

HRMS (TOF MS ES+) (m/z): calcd for $\mathrm{C}_{24} \mathrm{H}_{29} \mathrm{~N}_{5} \mathrm{O}_{3} \mathrm{Na}$ [M+Na]

\subsection{XRPD}

According to the literature, VAL may occur in 12 different polymorphs and an amorphous form which can be distinguished from each other with X-ray powder diffraction pattern.10 The XRPD pattern of VAL extracted from tablets and two literature forms are presented in Fig. S7. VAL used in this study is characterized by an XRPD pattern with broad amorphous halo together with several reflections at 12.60,14.84, 17.68, 22.19, 25.57, 27.23 which correspond to VAL form VII reflections described in the literature.10 So it can be concluded that VAL extracted from tablets occurs mostly in amorphous form with the addition of polymorphic form VII.

${ }^{9}$ N. Koseki, H. Kawashita, H. Hara, M. Niina, M. Tanaka, R. Kawai, Y. Nagae, N. Masuda, Development and validation of a method for quantitative determination of valsartan in human plasma by liquid chromatography-tandem mass spectrometry, J. Pharm. Biomed. Anal., 2007, 43, 1769-1774. 

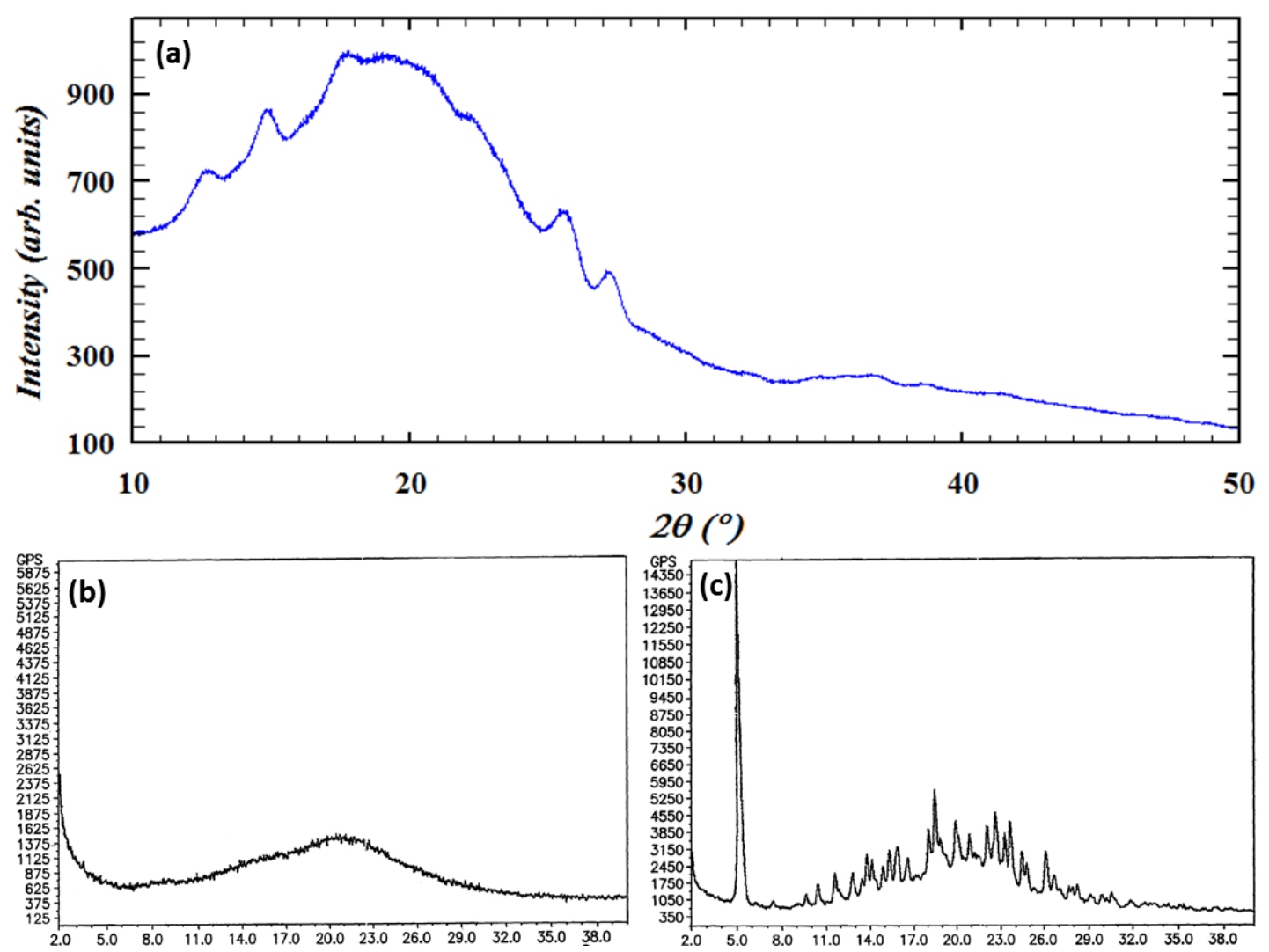

Fig. S7. XRPD pattern of (a) VAL used in this study; (b) VAL purely amorphous ${ }^{10}$; (c) VAL polymorphic form VII ${ }^{10}$.

\section{Nicotinamide (NIC)}

\subsection{XRPD}

The XRPD of the NIC sample used in this study can be accurately matched to the simulated XRPD pattern which was generated from the deposited X-ray data file at the Cambridge Structure Database (CSD - Refcode NICOAM06) using the Mercury software (Fig. S8).

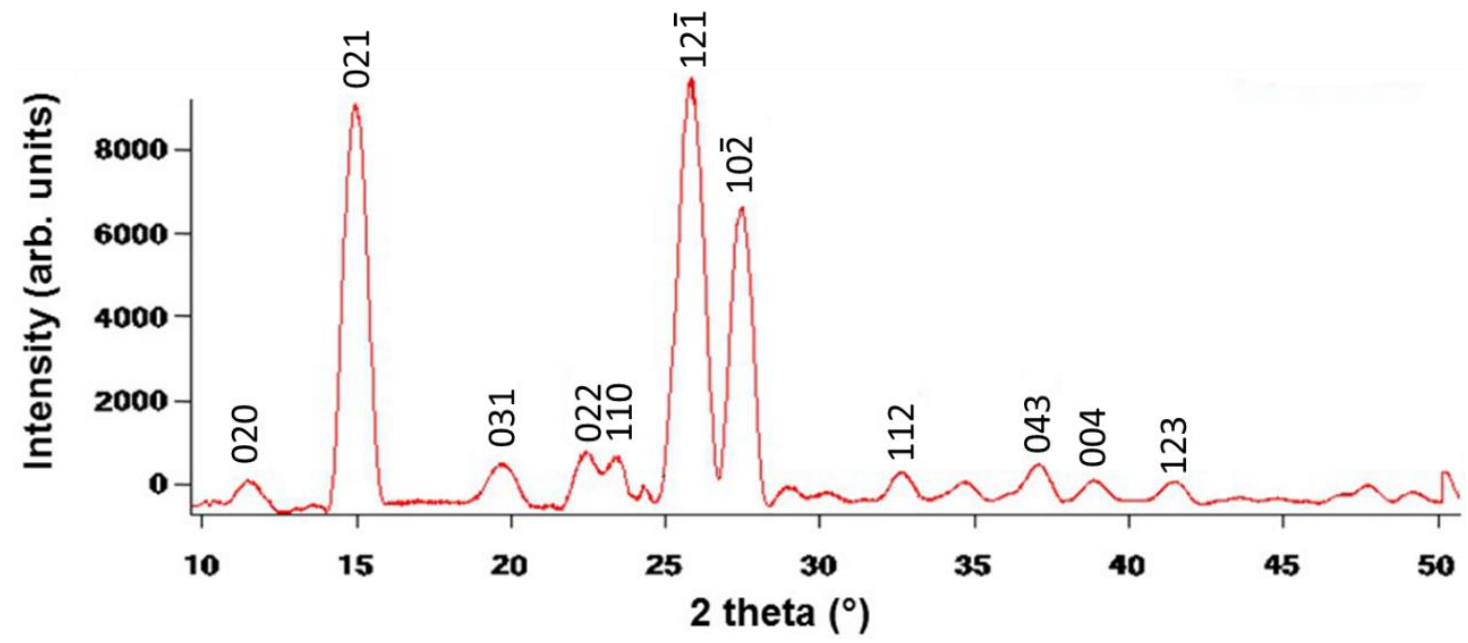

Fig. S8. XRPD pattern of NIC used in this study along with designation of major reflections. 


\section{Solid dispersions (VAL/NIC)}

\subsection{Solution-state NMR studies}

The solution-state ${ }^{1} \mathrm{H}$ NMR spectra $\left(\mathrm{CDCl}_{3}\right)$ were collected for co-amorphous solid dispersions to quantitatively estimate the amount of ethanol in obtained formulations. The spectra are presented in Fig. S8. The calculated composition of obtained materials is the following:

- VAL/NIC slurry -VAL 47.78\%, NIC 47.78\%, EtOH 4.43\% (by moles); VAL 77.51\%, NIC 21.73\%, EtOH 0.76\% (by weight);

- VAL/NIC LAG 30 min - VAL 49.26\%, NIC 49.26\%, EtOH 1.47\% (by moles); VAL 77.91\%, NIC 21.85\%, EtOH $0.25 \%$ (by weight);

- VAL/NIC LAG 60 min - VAL 47.14\%, NIC 47.14\%, EtOH 5.73\% (by moles); VAL 77.32\%, NIC 21.68\%, EtOH $0.99 \%$ (by weight);

- VAL/NIC ball mill - VAL 46.61\%, NIC 46.61\%, EtOH 6.79\% (by moles); VAL 77.17\%, NIC 21.64\%, EtOH 1.19\% (by weight).

${ }^{1} \mathrm{H}$ NMR spectra in $\mathrm{CDCl}_{3}$ show two valsartan rotamers ( $\mathrm{N}$ and $\mathrm{n}$ ) that differ from those mentioned in MeOD (Fig. S3a). Chashmniam and Tafazzoli reported valsartan's $\mathrm{n} / \mathrm{N}$ rotamers ratio in $\mathrm{CDCl}_{3}=0.22 .{ }^{8}$

The major/minor rotamers ratios in currently investigated solid dispersions are the following:

- VAL/NIC slurry - $1.7: 1.0(\mathrm{n} / \mathrm{N}=0.59)$

- VAL/NIC LAG 30 min - $2.6: 1.0(\mathrm{n} / \mathrm{N}=0.38)$

(a)

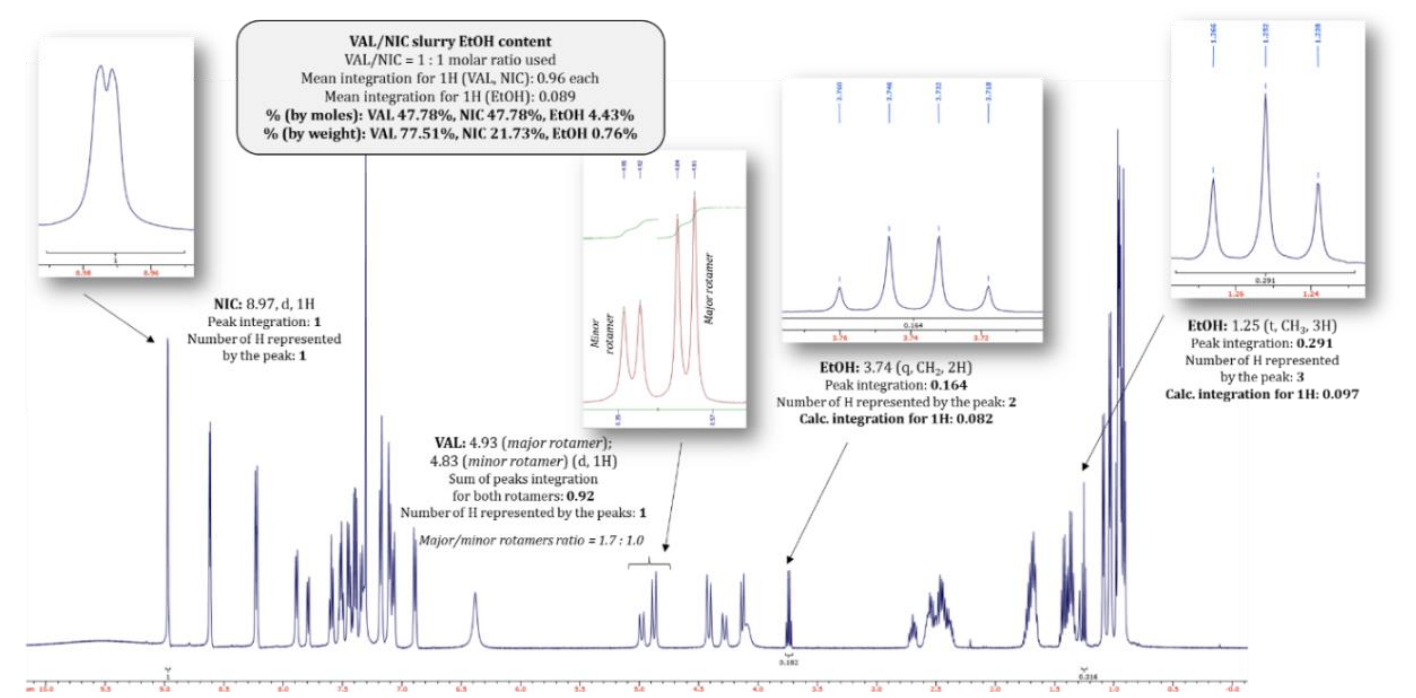

(b)

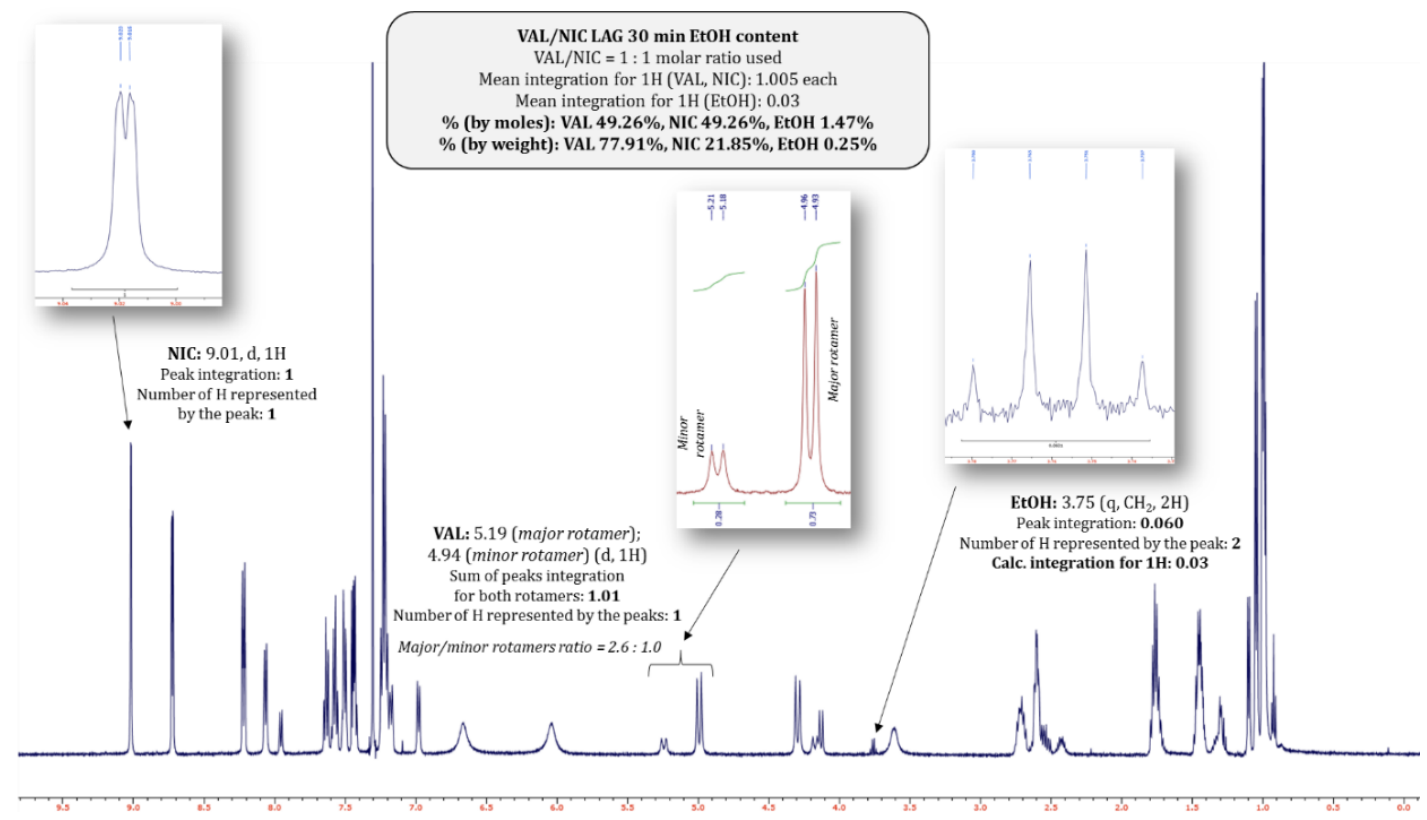

- VAL/NIC LAG 60 min - $2.0: 1.0(\mathrm{n} / \mathrm{N}=0.50)$

- VAL/NIC ball mill - $2.3: 1.0(\mathrm{n} / \mathrm{N}=0.43)$ 

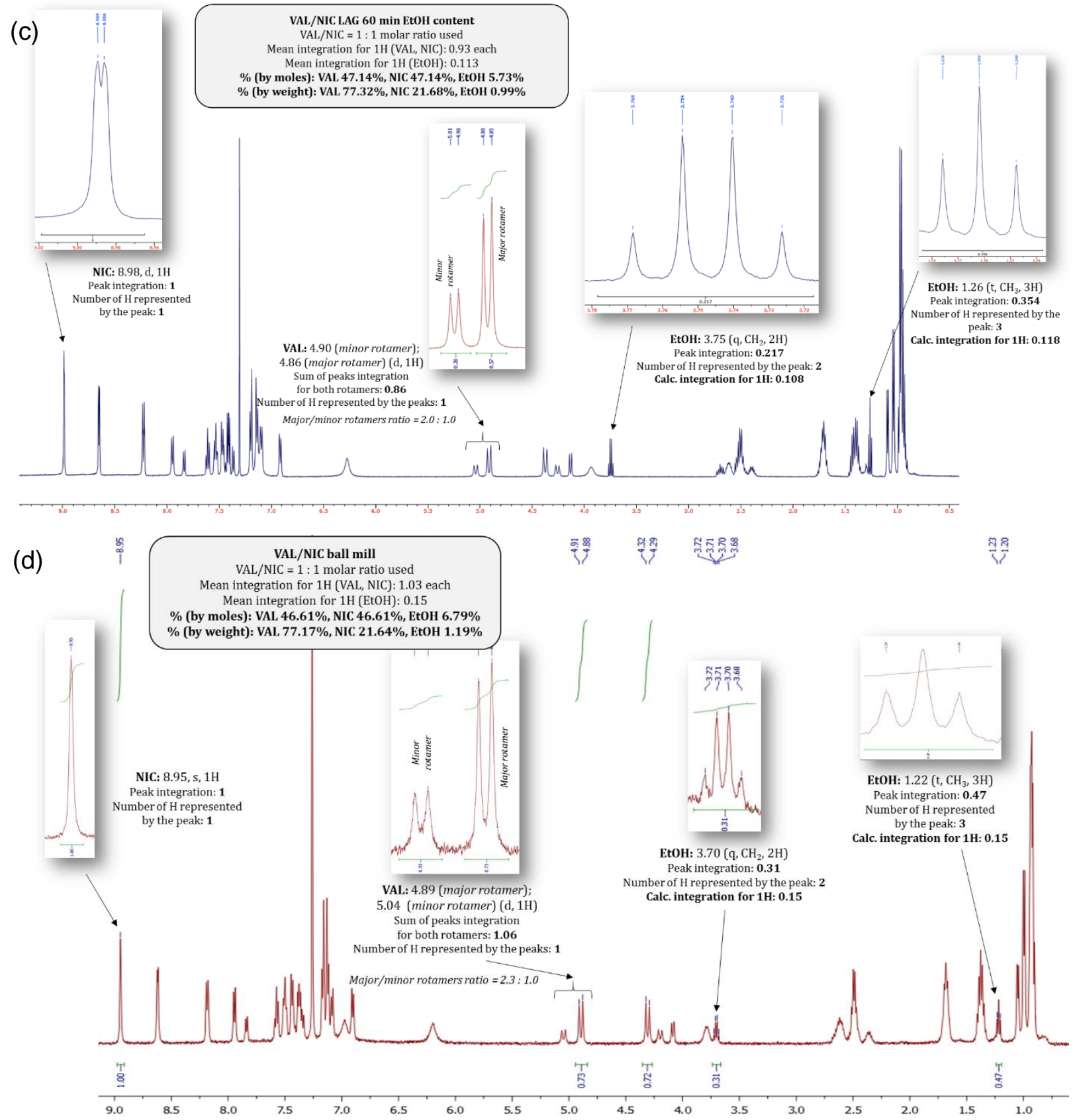

Fig. S9. ${ }^{1} \mathrm{H}$ NMR $\left(\mathrm{CDCl}_{3}\right)$ spectra for (a) VAL/NIC slurry, (b) VAL/NIC LAG $30 \mathrm{~min}$, (c) VAL/NIC LAG 60 min, (d) VAL/NIC ball mill along with calculations of the EtOH content. 


\section{Preliminary semi-empirical calculations}

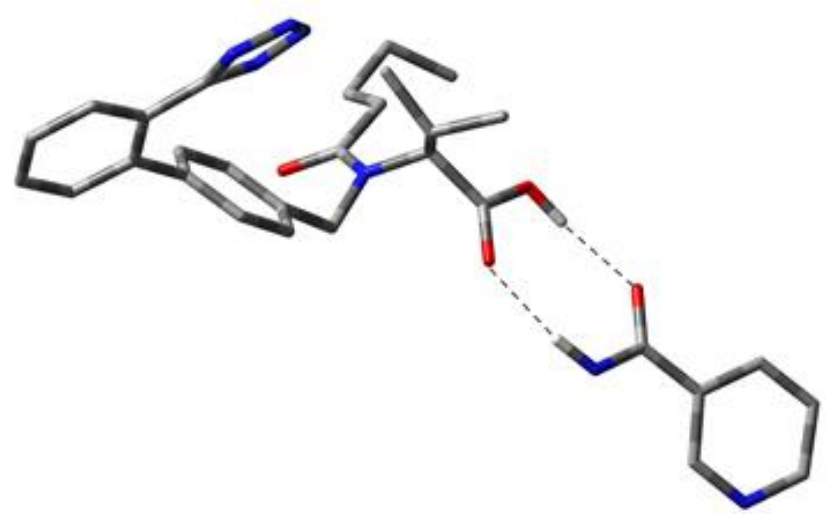

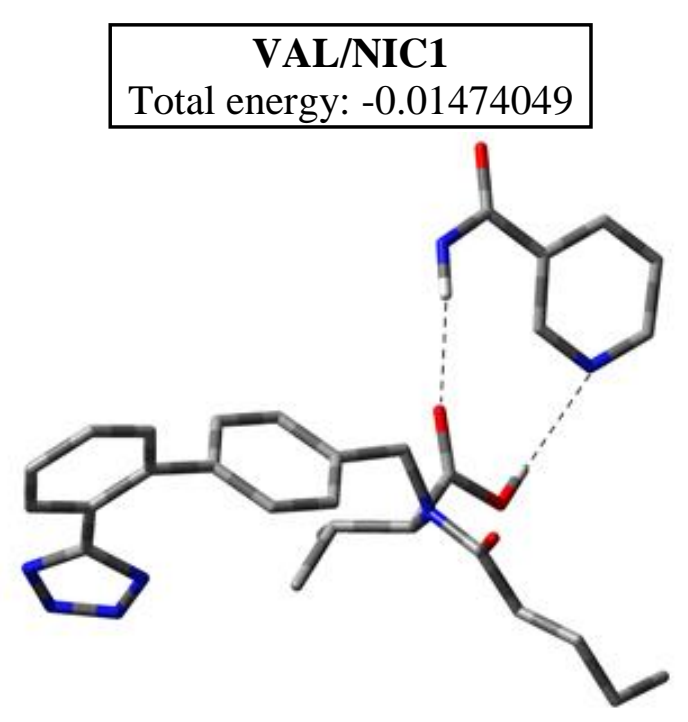

VAL/NIC3

Total energy: -0.01072872

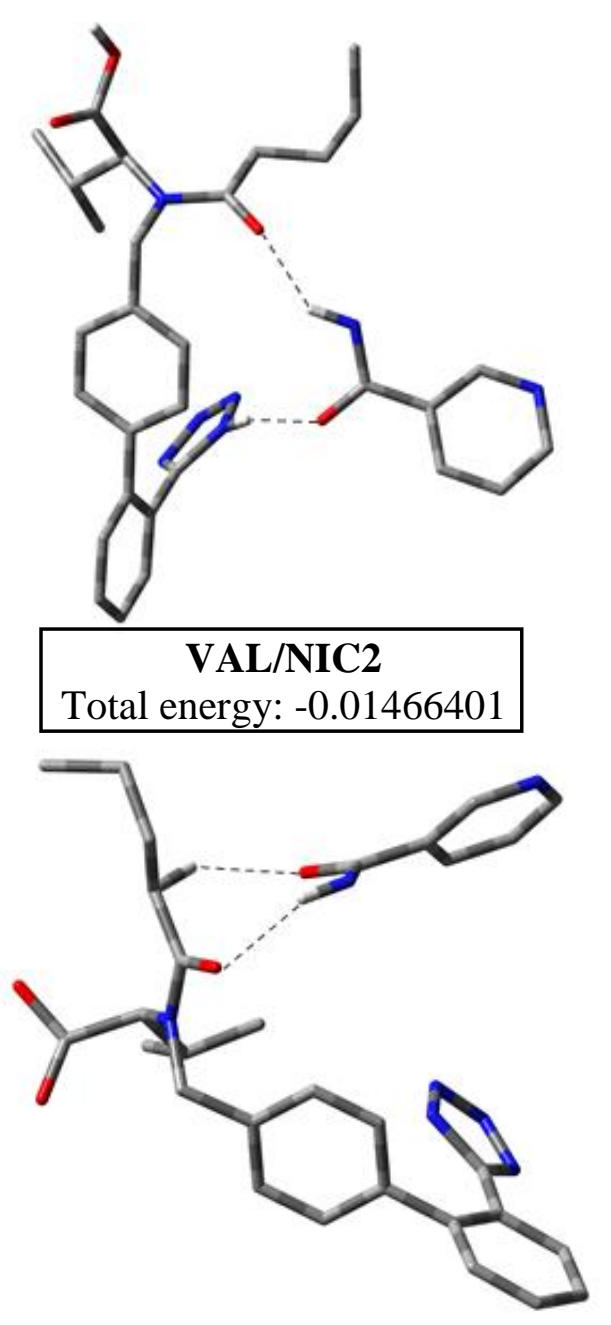

VAL/NIC4

Total energy: -0.01058827

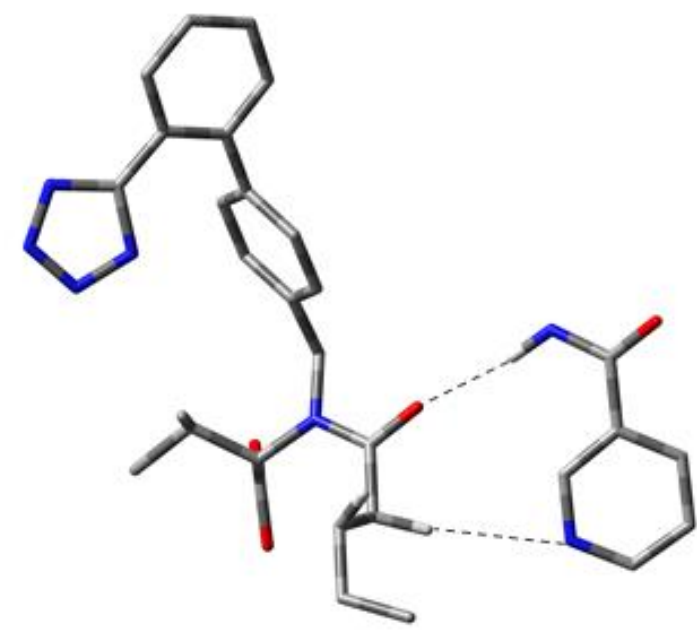

VAL/NIC5

Total energy: -0.01123121

Fig. S10. Chemical structures of 5 possible VAL/NIC heterodimers optimized by using AM1 method along with calculated total energy (Hartree). 


\section{DFT calculations}

\subsection{Cartesian coordinates and total energies for the optimized structures}

Table S2. Atom coordinates $(\AA)$, total energy (Hartree) and the number of imaginary vibrational frequencies for the geometry of VAL optimized at the B3LYP/6-311++(d,p) level in the gas phase using Gaussian 09.

\begin{tabular}{|c|c|c|c|}
\hline Atom & $\mathbf{X}$ & $\mathbf{Y}$ & $\mathbf{Z}$ \\
\hline 0 & -2.7684850 & -2.4280240 & -0.5030770 \\
\hline 0 & -4.9688980 & 1.5794580 & -1.4970850 \\
\hline $\mathrm{H}$ & -5.3945520 & 1.8758640 & -2.3173040 \\
\hline 0 & -3.1402920 & 2.1579170 & -2.6673180 \\
\hline $\mathrm{N}$ & 2.9384080 & 1.1781310 & 1.2818500 \\
\hline $\mathrm{H}$ & 2.1291850 & 0.6245730 & 1.0399720 \\
\hline $\mathrm{N}$ & -2.4567900 & -0.1953950 & -0.7193170 \\
\hline $\mathrm{N}$ & 2.8065650 & 2.3493290 & 1.9351560 \\
\hline $\mathrm{N}$ & 4.0017540 & 2.8172600 & 2.0718510 \\
\hline $\mathrm{N}$ & 4.9172680 & 1.9849780 & 1.5246770 \\
\hline $\mathrm{C}$ & 4.2465240 & 0.9555310 & 1.0245500 \\
\hline $\mathrm{C}$ & 4.8506680 & -0.2160610 & 0.3748400 \\
\hline $\mathrm{C}$ & 4.1286850 & -1.1295350 & -0.4237600 \\
\hline $\mathrm{C}$ & 2.6848860 & -0.9496460 & -0.7664370 \\
\hline $\mathrm{C}$ & 2.2806840 & 0.0319720 & -1.6787940 \\
\hline $\mathrm{H}$ & 3.0230590 & 0.6750980 & -2.1386440 \\
\hline $\mathrm{C}$ & 0.9340020 & 0.1908710 & -1.9987020 \\
\hline $\mathrm{H}$ & 0.6458840 & 0.9587390 & -2.7097350 \\
\hline $\mathrm{C}$ & -0.0479470 & -0.6214580 & -1.4232470 \\
\hline $\mathrm{C}$ & -1.5031680 & -0.4500070 & -1.8230730 \\
\hline $\mathrm{H}$ & -1.5848220 & 0.3718810 & -2.5333660 \\
\hline $\mathrm{H}$ & -1.8420010 & -1.3600440 & -2.3214920 \\
\hline $\mathrm{C}$ & -3.0951540 & -1.2967750 & -0.1706330 \\
\hline $\mathrm{C}$ & -4.1982210 & -1.0656280 & 0.8610490 \\
\hline $\mathrm{H}$ & -4.9806410 & -0.4495160 & 0.4078120 \\
\hline $\mathrm{H}$ & -3.7910490 & -0.4804830 & 1.6949360 \\
\hline $\mathrm{C}$ & -4.7866400 & -2.3753890 & 1.3947050 \\
\hline $\mathrm{H}$ & -3.9737230 & -2.9969220 & 1.7788290 \\
\hline $\mathrm{H}$ & -5.2239960 & -2.9384480 & 0.5643000 \\
\hline $\mathrm{C}$ & -5.8377730 & -2.1699640 & 2.4957720 \\
\hline $\mathrm{H}$ & -6.1036290 & -3.1529540 & 2.8992770 \\
\hline $\mathrm{H}$ & -5.3895770 & -1.6147110 & 3.3296310 \\
\hline $\mathrm{C}$ & -7.1176950 & -1.4603180 & 2.0387080 \\
\hline $\mathrm{H}$ & -6.9255250 & -0.4351150 & 1.7098640 \\
\hline $\mathrm{H}$ & -7.8483930 & -1.4087520 & 2.8504620 \\
\hline $\mathrm{H}$ & -7.5861090 & -1.9934880 & 1.2049580 \\
\hline $\mathrm{C}$ & 1.7032670 & -1.7780520 & -0.2040420 \\
\hline $\mathrm{H}$ & 1.9983910 & -2.5553360 & 0.4928750 \\
\hline $\mathrm{C}$ & 0.3572390 & -1.6183210 & -0.5277980 \\
\hline $\mathrm{H}$ & -0.3858440 & -2.2787860 & -0.0978260 \\
\hline $\mathrm{C}$ & 6.2197620 & -0.4279240 & 0.6043990 \\
\hline $\mathrm{H}$ & 6.7556240 & 0.2862730 & 1.2164440 \\
\hline $\mathrm{C}$ & 6.8690490 & -1.5323840 & 0.0709270 \\
\hline $\mathrm{H}$ & 7.9256710 & -1.6800980 & 0.2611530 \\
\hline $\mathrm{C}$ & 6.1556720 & -2.4499970 & -0.6982060 \\
\hline $\mathrm{H}$ & 6.6519030 & -3.3191620 & -1.1147450 \\
\hline $\mathrm{C}$ & 4.8010830 & -2.2439340 & -0.9395240 \\
\hline $\mathrm{H}$ & 4.2480400 & -2.9452850 & -1.5538880 \\
\hline
\end{tabular}




\begin{tabular}{|c|c|c|c|}
\hline $\mathrm{C}$ & -2.8612560 & 1.1957770 & -0.4370990 \\
\hline $\mathrm{H}$ & -3.5643530 & 1.1689970 & 0.3909030 \\
\hline $\mathrm{C}$ & -3.6308990 & 1.7172030 & -1.6580800 \\
\hline $\mathrm{C}$ & -1.7009510 & 2.1320370 & -0.0308550 \\
\hline $\mathrm{H}$ & -0.9702140 & 2.1459390 & -0.8445350 \\
\hline $\mathrm{C}$ & -2.2125760 & 3.5670770 & 0.1731850 \\
\hline $\mathrm{H}$ & -2.9853710 & 3.6028650 & 0.9491180 \\
\hline $\mathrm{H}$ & -2.6245730 & 3.9933650 & -0.7437770 \\
\hline $\mathrm{H}$ & -1.3935660 & 4.2130590 & 0.4975700 \\
\hline $\mathrm{C}$ & -1.0207830 & 1.6235050 & 1.2471300 \\
\hline $\mathrm{H}$ & -0.1615670 & 2.2507890 & 1.4978270 \\
\hline $\mathrm{H}$ & -0.6770970 & 0.5928060 & 1.1424730 \\
\hline $\mathrm{H}$ & -1.7109370 & 1.6624420 & 2.0971520 \\
\hline \multicolumn{2}{|c|}{ Total energy } & \multicolumn{2}{|c|}{-1431.83718410} \\
\hline \multicolumn{2}{|c|}{ Number of imaginary vibrational frequencies } & \multicolumn{2}{|c|}{0} \\
\hline
\end{tabular}

Table S3. Atom coordinates $(\AA)$, total energy (Hartree) and the number of imaginary vibrational frequencies for the geometry of NIC optimized at the B3LYP/6-311++(d,p) level in the gas phase using Gaussian 09.

\begin{tabular}{|c|c|c|c|}
\hline Atom & $\mathbf{X}$ & $\mathbf{Y}$ & $\mathbf{Z}$ \\
\hline $\mathrm{C}$ & 1.7046700 & 0.1438590 & -0.0298970 \\
\hline $\mathrm{N}$ & 2.4042190 & -1.0056210 & 0.2221040 \\
\hline $\mathrm{O}$ & 2.2627750 & 1.2006900 & -0.2746680 \\
\hline $\mathrm{H}$ & 3.4073790 & -0.9208750 & 0.2850020 \\
\hline $\mathrm{C}$ & 1.9733640 & -1.8069080 & 0.6521700 \\
\hline $\mathrm{C}$ & 0.2057170 & 0.0343940 & -0.0103280 \\
\hline $\mathrm{C}$ & -0.4915110 & -1.1705240 & -0.1493110 \\
\hline $\mathrm{H}$ & -0.5423180 & 1.2070130 & 0.1226570 \\
\hline $\mathrm{C}$ & 0.0387280 & -2.1064230 & -0.3046240 \\
\hline $\mathrm{H}$ & -1.9274670 & 1.1214020 & 0.1391840 \\
\hline $\mathrm{H}$ & -0.0234880 & 2.1542570 & 0.2067700 \\
\hline $\mathrm{N}$ & -2.5427080 & 2.0066600 & 0.2472230 \\
\hline $\mathrm{H}$ & -1.8231190 & -1.2655150 & -0.1387610 \\
\hline \multicolumn{2}{|}{ Total energy } & -0.1352910 & 0.0114710 \\
\hline & -2.5195110 & -0.2393950 & 0.0247490 \\
\hline
\end{tabular}

Table S4a. Atom coordinates $(\AA)$, total energy (Hartree) and the number of imaginary vibrational frequencies for the geometry of VAL/NIC1 optimized at the B3LYP/6-311++(d,p) level in the gas phase using Gaussian 09.

\begin{tabular}{|c|c|c|c|}
\hline Atom & $\mathbf{X}$ & $\mathbf{Y}$ & $\mathbf{Z}$ \\
\hline $\mathrm{O}$ & 0.0916940 & 2.7215260 & -2.0646030 \\
\hline $\mathrm{O}$ & 3.3184970 & 0.9107470 & 0.8369690 \\
\hline $\mathrm{H}$ & 4.1610180 & 0.3989040 & 0.6350960 \\
\hline $\mathrm{O}$ & 2.2791030 & -1.0535550 & 0.4903180 \\
\hline $\mathrm{N}$ & -4.9289920 & -0.4874130 & 1.6536810 \\
\hline $\mathrm{H}$ & -4.2580300 & 0.0505370 & 1.1243090 \\
\hline $\mathrm{N}$ & 0.3874900 & 1.2620780 & -0.3588480 \\
\hline $\mathrm{N}$ & -4.7940730 & -0.6432530 & 2.9853530 \\
\hline $\mathrm{N}$ & -5.7764920 & -1.3930280 & 3.3574680 \\
\hline $\mathrm{N}$ & -6.5546150 & -1.7364050 & 2.3047570 \\
\hline $\mathrm{C}$ & -6.0176190 & -1.1691140 & 1.2328870 \\
\hline $\mathrm{C}$ & -6.5668400 & -1.2348910 & -0.1286650 \\
\hline $\mathrm{C}$ & -5.8144500 & -0.9581430 & -1.2910120 \\
\hline
\end{tabular}




\begin{tabular}{|c|c|c|c|}
\hline $\mathrm{C}$ & -4.3503620 & -0.6623770 & -1.2636670 \\
\hline $\mathrm{C}$ & -3.4218180 & -1.6574890 & -0.9360400 \\
\hline $\mathrm{H}$ & -3.7694130 & -2.6552850 & -0.6912140 \\
\hline $\mathrm{C}$ & -2.0575550 & -1.3763530 & -0.9207140 \\
\hline $\mathrm{H}$ & -1.3576160 & -2.1650500 & -0.6633890 \\
\hline $\mathrm{C}$ & -1.5763530 & -0.1005190 & -1.2309920 \\
\hline $\mathrm{C}$ & -0.0810480 & 0.1668640 & -1.2370880 \\
\hline $\mathrm{H}$ & 0.4478410 & -0.7402580 & -0.9480350 \\
\hline $\mathrm{H}$ & 0.2264780 & 0.4288380 & -2.2512010 \\
\hline $\mathrm{C}$ & 0.5031070 & 2.5189090 & -0.9292560 \\
\hline $\mathrm{C}$ & 1.1419970 & 3.6397180 & -0.1109880 \\
\hline $\mathrm{H}$ & 2.1491390 & 3.3294410 & 0.1818210 \\
\hline $\mathrm{H}$ & 0.5773220 & 3.7688850 & 0.8206810 \\
\hline $\mathrm{C}$ & 1.1859190 & 4.9690540 & -0.8701920 \\
\hline $\mathrm{H}$ & 0.1735350 & 5.2252840 & -1.1931690 \\
\hline $\mathrm{H}$ & 1.7669180 & 4.8396550 & -1.7885480 \\
\hline $\mathrm{C}$ & 1.7687450 & 6.1256150 & -0.0437730 \\
\hline $\mathrm{H}$ & 1.6302460 & 7.0527650 & -0.6105450 \\
\hline $\mathrm{H}$ & 1.1846960 & 6.2470090 & 0.8775810 \\
\hline $\mathrm{C}$ & 3.2539610 & 5.9785840 & 0.3075840 \\
\hline $\mathrm{H}$ & 3.4431100 & 5.1139660 & 0.9499180 \\
\hline $\mathrm{H}$ & 3.6208010 & 6.8624660 & 0.8367890 \\
\hline $\mathrm{H}$ & 3.8603810 & 5.8578410 & -0.5959300 \\
\hline $\mathrm{C}$ & -3.8679480 & 0.6114440 & -1.5965560 \\
\hline $\mathrm{H}$ & -4.5704730 & 1.3920540 & -1.8688450 \\
\hline $\mathrm{C}$ & -2.5025530 & 0.8889390 & -1.5822170 \\
\hline $\mathrm{H}$ & -2.1460690 & 1.8738360 & -1.8582130 \\
\hline $\mathrm{C}$ & -7.9271230 & -1.5630060 & -0.2442630 \\
\hline $\mathrm{H}$ & -8.4843200 & -1.7753950 & 0.6592690 \\
\hline $\mathrm{C}$ & -8.5477510 & -1.6008970 & -1.4851260 \\
\hline $\mathrm{H}$ & -9.5992780 & -1.8537560 & -1.5543880 \\
\hline $\mathrm{C}$ & -7.8164250 & -1.3032560 & -2.6336070 \\
\hline $\mathrm{H}$ & -8.2927210 & -1.3240190 & -3.6072320 \\
\hline $\mathrm{C}$ & -6.4654690 & -0.9875740 & -2.5303920 \\
\hline $\mathrm{H}$ & -5.8891970 & -0.7778910 & -3.4243350 \\
\hline $\mathrm{C}$ & 0.9430730 & 0.9226220 & 0.9676690 \\
\hline $\mathrm{H}$ & 1.2205890 & 1.8557300 & 1.4504330 \\
\hline $\mathrm{C}$ & 2.2477620 & 0.1402950 & 0.7514630 \\
\hline $\mathrm{C}$ & -0.0455950 & 0.1930640 & 1.9042080 \\
\hline $\mathrm{H}$ & -0.3610370 & -0.7340500 & 1.4170350 \\
\hline C & 0.6420470 & -0.1781580 & 3.2281200 \\
\hline $\mathrm{H}$ & 1.0206010 & 0.7137240 & 3.7394080 \\
\hline $\mathrm{H}$ & 1.4758210 & -0.8676240 & 3.0802800 \\
\hline $\mathrm{H}$ & -0.0724200 & -0.6620570 & 3.8982260 \\
\hline $\mathrm{C}$ & -1.2800700 & 1.0633320 & 2.1729970 \\
\hline $\mathrm{H}$ & -2.0003930 & 0.5222990 & 2.7921940 \\
\hline $\mathrm{H}$ & -1.7748700 & 1.3628220 & 1.2473390 \\
\hline $\mathrm{H}$ & -1.0030890 & 1.9742120 & 2.7152900 \\
\hline $\mathrm{C}$ & 5.8005660 & -1.5043180 & 0.0215810 \\
\hline $\mathrm{N}$ & 4.8002180 & -2.4001590 & -0.0605900 \\
\hline 0 & 5.5987750 & -0.3153530 & 0.3101840 \\
\hline $\mathrm{H}$ & 3.8468410 & -2.0786190 & 0.1191900 \\
\hline $\mathrm{H}$ & 4.9779810 & -3.3812660 & -0.1920550 \\
\hline $\mathrm{C}$ & 7.1964120 & -1.9737200 & -0.2463120 \\
\hline $\mathrm{C}$ & 7.4966800 & -3.1467920 & -0.9476810 \\
\hline
\end{tabular}




\begin{tabular}{|c|c|c|c|}
\hline $\mathrm{C}$ & 8.2640830 & -1.1890800 & 0.1981670 \\
\hline $\mathrm{H}$ & 6.7073790 & -3.7708410 & -1.3590220 \\
\hline $\mathrm{C}$ & 9.5602250 & -1.6194600 & -0.0482240 \\
\hline $\mathrm{H}$ & 8.0570150 & -0.2644420 & 0.7224700 \\
\hline $\mathrm{H}$ & 10.4147160 & -1.0436990 & 0.2862670 \\
\hline $\mathrm{C}$ & 8.7379510 & -3.5680260 & -1.1938080 \\
\hline $\mathrm{H}$ & 9.7453640 & -2.8157410 & -0.7411060 \\
\hline \multicolumn{2}{|c|}{ Total energy } & -3.1848090 & -0.9473280 \\
\hline \multicolumn{2}{|c|}{0} \\
\hline
\end{tabular}

Table S4b. Atom coordinates $(\AA)$, total energy (Hartree) and the number of imaginary vibrational frequencies for the geometry of VAL/NIC1 optimized at the B3LYP/6-311++(d,p) level in ethanol using Gaussian 09.

\begin{tabular}{|c|c|c|c|}
\hline Atom & $\mathbf{X}$ & $Y$ & $\mathbf{Z}$ \\
\hline 0 & 0.1789950 & 2.8987800 & -1.8287450 \\
\hline 0 & 3.3726180 & 0.7996950 & 0.9166200 \\
\hline $\mathrm{H}$ & 4.1987480 & 0.2807480 & 0.6582710 \\
\hline 0 & 2.2711650 & -1.0716450 & 0.3414150 \\
\hline $\mathrm{N}$ & -5.1163990 & -1.0441820 & 1.7132840 \\
\hline $\mathrm{H}$ & -4.5745290 & -0.2178300 & 1.4974410 \\
\hline $\mathrm{N}$ & 0.4040940 & 1.3289220 & -0.2119180 \\
\hline $\mathrm{N}$ & -4.9089590 & -1.7234550 & 2.8529140 \\
\hline $\mathrm{N}$ & -5.7381470 & -2.7136670 & 2.8337010 \\
\hline $\mathrm{N}$ & -6.4858930 & -2.7021340 & 1.7057800 \\
\hline $\mathrm{C}$ & -6.0890600 & -1.6489660 & 1.0002370 \\
\hline $\mathrm{C}$ & -6.6653960 & -1.2002440 & -0.2743720 \\
\hline $\mathrm{C}$ & -5.9198350 & -0.5278140 & -1.2678560 \\
\hline $\mathrm{C}$ & -4.4469170 & -0.3076790 & -1.1808480 \\
\hline $\mathrm{C}$ & -3.5568850 & -1.3851240 & -1.0866480 \\
\hline $\mathrm{H}$ & -3.9386730 & -2.3994900 & -1.0493990 \\
\hline $\mathrm{C}$ & -2.1814070 & -1.1683460 & -1.0525870 \\
\hline $\mathrm{H}$ & -1.5140510 & -2.0214120 & -0.9839880 \\
\hline $\mathrm{C}$ & -1.6494440 & 0.1238220 & -1.1145510 \\
\hline $\mathrm{C}$ & -0.1422830 & 0.3128120 & -1.1374880 \\
\hline $\mathrm{H}$ & 0.3417860 & -0.6359940 & -0.9140560 \\
\hline $\mathrm{H}$ & 0.1611820 & 0.6070590 & -2.1439450 \\
\hline $\mathrm{C}$ & 0.5773950 & 2.6062750 & -0.7014690 \\
\hline $\mathrm{C}$ & 1.2553880 & 3.6465990 & 0.1860060 \\
\hline $\mathrm{H}$ & 2.2680850 & 3.3008120 & 0.4130520 \\
\hline $\mathrm{H}$ & 0.7279110 & 3.6966040 & 1.1452350 \\
\hline $\mathrm{C}$ & 1.2933180 & 5.0412170 & -0.4454980 \\
\hline $\mathrm{H}$ & 0.2724790 & 5.3466340 & -0.6918730 \\
\hline $\mathrm{H}$ & 1.8365420 & 4.9974250 & -1.3948140 \\
\hline $\mathrm{C}$ & 1.9319830 & 6.1030790 & 0.4633330 \\
\hline $\mathrm{H}$ & 1.7750150 & 7.0845270 & 0.0027960 \\
\hline $\mathrm{H}$ & 1.3993780 & 6.1295120 & 1.4220890 \\
\hline $\mathrm{C}$ & 3.4319780 & 5.9098020 & 0.7153500 \\
\hline $\mathrm{H}$ & 3.6431380 & 4.9792050 & 1.2494850 \\
\hline $\mathrm{H}$ & 3.8356290 & 6.7292800 & 1.3166500 \\
\hline $\mathrm{H}$ & 3.9870670 & 5.8850140 & -0.2281240 \\
\hline $\mathrm{C}$ & -3.9142890 & 0.9876670 & -1.2482270 \\
\hline $\mathrm{H}$ & -4.5839780 & 1.8373100 & -1.3237730 \\
\hline $\mathrm{C}$ & -2.5382760 & 1.1997920 & -1.2155940 \\
\hline $\mathrm{H}$ & -2.1528900 & 2.2095880 & -1.2783900 \\
\hline $\mathrm{C}$ & -8.0365460 & -1.4322160 & -0.4641810 \\
\hline
\end{tabular}




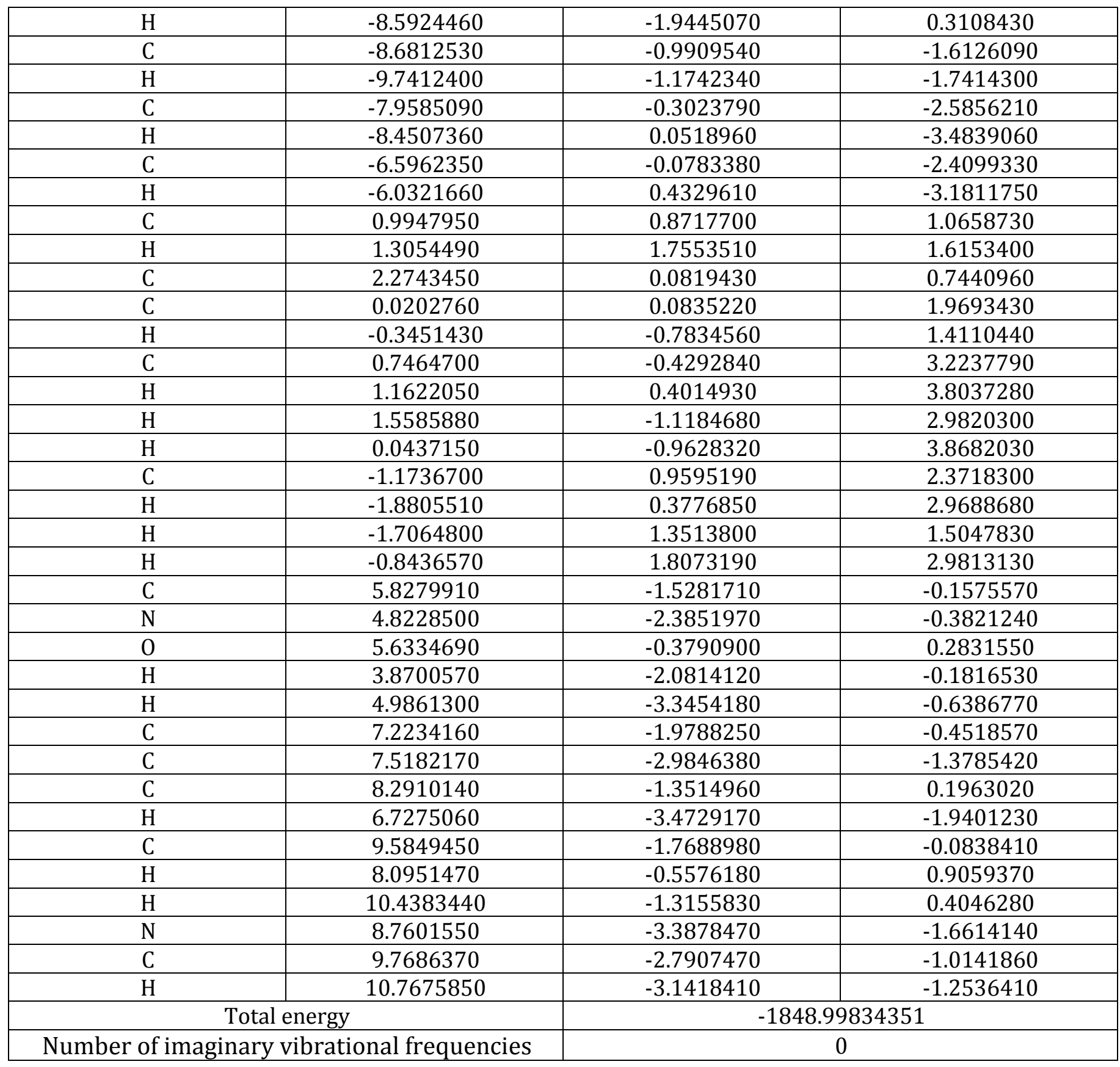

Table S5a. Atom coordinates $(\AA)$, total energy (Hartree) and the number of imaginary vibrational frequencies for the geometry of VAL/NIC2 optimized at the B3LYP/6-311++(d,p) level in the gas phase using Gaussian 09.

\begin{tabular}{|c|c|c|c|}
\hline Atom & $\mathbf{X}$ & $\mathbf{Y}$ & $\mathbf{Z}$ \\
\hline $\mathrm{O}$ & 1.5577490 & 1.5359160 & -0.8428210 \\
\hline $\mathrm{O}$ & 6.0016830 & 1.0442570 & -1.5588140 \\
\hline $\mathrm{H}$ & 6.5142280 & 1.1464500 & -2.3763780 \\
\hline $\mathrm{O}$ & 5.6347020 & -0.9774440 & -2.4690790 \\
\hline $\mathrm{N}$ & -1.8477440 & -1.1229230 & 1.8864450 \\
\hline $\mathrm{H}$ & -1.9554200 & -0.3167770 & 1.2590190 \\
\hline $\mathrm{N}$ & 3.1784990 & -0.0269350 & -0.7351790 \\
\hline $\mathrm{N}$ & -1.1804150 & -1.0029770 & 3.0468900 \\
\hline $\mathrm{N}$ & -1.2751240 & -2.1450030 & 3.6451580 \\
\hline $\mathrm{N}$ & -1.9982760 & -3.0218080 & 2.9048250 \\
\hline $\mathrm{C}$ & -2.3474400 & -2.3723800 & 1.8045770 \\
\hline $\mathrm{C}$ & -3.1631690 & -2.9100710 & 0.6999330 \\
\hline
\end{tabular}




\begin{tabular}{|c|c|c|c|}
\hline $\mathrm{C}$ & -2.7327230 & -2.8618820 & -0.6433000 \\
\hline $\mathrm{C}$ & -1.3815850 & -2.3575090 & -1.0122400 \\
\hline $\mathrm{C}$ & -0.2306810 & -2.9091250 & -0.4345380 \\
\hline $\mathrm{H}$ & -0.3225030 & -3.7394490 & 0.2563520 \\
\hline $\mathrm{C}$ & 1.0281720 & -2.4006020 & -0.7336110 \\
\hline $\mathrm{H}$ & 1.8978100 & -2.8548190 & -0.2743710 \\
\hline $\mathrm{C}$ & 1.1782430 & -1.3143500 & -1.6005690 \\
\hline $\bar{C}$ & 2.5411020 & -0.7140540 & -1.8901630 \\
\hline $\mathrm{H}$ & 3.2308830 & -1.4924310 & -2.2136380 \\
\hline $\mathrm{H}$ & 2.4494080 & 0.0014460 & -2.7111050 \\
\hline $\mathrm{C}$ & 2.5810300 & 1.1307910 & -0.2938780 \\
\hline C & 3.1930190 & 1.8807750 & 0.8861650 \\
\hline $\mathrm{H}$ & 4.1394370 & 2.3214580 & 0.5534380 \\
\hline $\mathrm{H}$ & 3.4429620 & 1.1699940 & 1.6803220 \\
\hline $\mathrm{C}$ & 2.2688210 & 2.9666770 & 1.4462510 \\
\hline $\mathrm{H}$ & 1.3226550 & 2.5056600 & 1.7428520 \\
\hline $\mathrm{H}$ & 2.0274580 & 3.6809070 & 0.6531790 \\
\hline $\mathrm{C}$ & 2.8631850 & 3.7131670 & 2.6505690 \\
\hline $\mathrm{H}$ & 2.0802500 & 4.3514440 & 3.0734200 \\
\hline $\mathrm{H}$ & 3.1186970 & 2.9930560 & 3.4374600 \\
\hline $\mathrm{C}$ & 4.0864340 & 4.5788970 & 2.3272320 \\
\hline $\mathrm{H}$ & 4.9379390 & 3.9828320 & 1.9863810 \\
\hline $\mathrm{H}$ & 4.4153470 & 5.1358550 & 3.2087010 \\
\hline $\mathrm{H}$ & 3.8561180 & 5.3069730 & 1.5425870 \\
\hline $\mathrm{C}$ & -1.2255530 & -1.3168480 & -1.9340400 \\
\hline $\mathrm{H}$ & -2.1018640 & -0.8745070 & -2.3942210 \\
\hline $\mathrm{C}$ & 0.0353580 & -0.7995870 & -2.2149750 \\
\hline $\mathrm{H}$ & 0.1295680 & 0.0429290 & -2.8904130 \\
\hline $\mathrm{C}$ & -4.4082470 & -3.4674610 & 1.0146920 \\
\hline $\mathrm{H}$ & -4.7126240 & -3.5096970 & 2.0535170 \\
\hline $\mathrm{C}$ & -5.2458080 & -3.9465490 & 0.0134430 \\
\hline $\mathrm{H}$ & -6.2079330 & -4.3735990 & 0.2717710 \\
\hline $\mathrm{C}$ & -4.8428050 & -3.8695970 & -1.3182460 \\
\hline $\mathrm{H}$ & -5.4889810 & -4.2393680 & -2.1065440 \\
\hline $\mathrm{C}$ & -3.5978760 & -3.3357220 & -1.6375980 \\
\hline $\mathrm{H}$ & -3.2698450 & -3.3111680 & -2.6707270 \\
\hline $\mathrm{C}$ & 4.5512540 & -0.4136680 & -0.3484290 \\
\hline $\mathrm{H}$ & 4.8858300 & 0.2931940 & 0.4063760 \\
\hline $\mathrm{C}$ & 5.4516870 & -0.1936470 & -1.5730780 \\
\hline $\mathrm{C}$ & 4.6769710 & -1.8331870 & 0.2471040 \\
\hline $\mathrm{H}$ & 4.2698130 & -2.5456490 & -0.4778920 \\
\hline $\mathrm{C}$ & 6.1505750 & -2.1987210 & 0.4892520 \\
\hline $\mathrm{H}$ & 6.6297030 & -1.4781760 & 1.1614580 \\
\hline $\mathrm{H}$ & 6.7232630 & -2.2412610 & -0.4385310 \\
\hline $\mathrm{H}$ & 6.2167480 & -3.1801470 & 0.9647950 \\
\hline $\mathrm{C}$ & 3.8847720 & -1.9324120 & 1.5584910 \\
\hline $\mathrm{H}$ & 3.8796420 & -2.9626860 & 1.9230970 \\
\hline $\mathrm{H}$ & 2.8477380 & -1.6135710 & 1.4463150 \\
\hline $\mathrm{H}$ & 4.3462570 & -1.3154590 & 2.3369030 \\
\hline $\mathrm{C}$ & -2.3112690 & 2.2191540 & -0.2462460 \\
\hline $\mathrm{N}$ & -1.1243720 & 2.6693900 & -0.6969740 \\
\hline 0 & -2.4459230 & 1.1345350 & 0.3247750 \\
\hline $\mathrm{H}$ & -0.2730380 & 2.1179300 & -0.5990330 \\
\hline $\mathrm{H}$ & -1.0642080 & 3.5051810 & -1.2535200 \\
\hline $\mathrm{C}$ & -3.5106280 & 3.0981110 & -0.4690240 \\
\hline
\end{tabular}




\begin{tabular}{|c|c|c|c|}
\hline $\mathrm{C}$ & -3.4338060 & 4.4695750 & -0.7362070 \\
\hline $\mathrm{C}$ & -4.7822100 & 2.5267140 & -0.3755070 \\
\hline $\mathrm{H}$ & -2.4746660 & 4.9796870 & -0.7730800 \\
\hline $\mathrm{C}$ & -5.8969180 & 3.3283900 & -0.5791950 \\
\hline $\mathrm{H}$ & -4.8697150 & 1.4723320 & -0.1442950 \\
\hline $\mathrm{H}$ & -6.8987600 & 2.9199400 & -0.5218320 \\
\hline $\mathrm{C}$ & -4.4974390 & 5.2519350 & -0.9293750 \\
\hline $\mathrm{H}$ & -5.7034440 & 4.6809110 & -0.8585470 \\
\hline \multicolumn{2}{|c|}{ Total energy } & 5.3368900 & -1.0273060 \\
\hline Number of imaginary vibrational frequencies & \multicolumn{2}{|c|}{0} \\
\hline
\end{tabular}

Table S5b. Atom coordinates $(\AA)$, total energy (Hartree) and the number of imaginary vibrational frequencies for the geometry of VAL/NIC2 optimized at the B3LYP/6-311++(d,p) level in ethanol using Gaussian 09.

\begin{tabular}{|c|c|c|c|}
\hline Atom & $\mathbf{X}$ & $\mathbf{Y}$ & $\mathbf{Z}$ \\
\hline 0 & 1.5195280 & 1.2771300 & -0.6289240 \\
\hline 0 & 5.9237050 & 0.9925450 & -1.6668570 \\
\hline $\mathrm{H}$ & 6.3630020 & 1.1253750 & -2.5233970 \\
\hline 0 & 5.5518250 & -1.0459680 & -2.5347090 \\
\hline $\mathrm{N}$ & -2.3053050 & -0.8737060 & 1.7672900 \\
\hline $\mathrm{H}$ & -2.2977880 & -0.1886230 & 0.9943840 \\
\hline $\mathrm{N}$ & 3.2192040 & -0.2027440 & -0.6006740 \\
\hline $\mathrm{N}$ & -1.8760460 & -0.5067400 & 2.9833570 \\
\hline $\mathrm{N}$ & -2.0627500 & -1.5225280 & 3.7625620 \\
\hline $\mathrm{N}$ & -2.6118760 & -2.5562500 & 3.0831190 \\
\hline $\mathrm{C}$ & -2.7580280 & -2.1408970 & 1.8299070 \\
\hline $\mathrm{C}$ & -3.3662160 & -2.9168590 & 0.7341970 \\
\hline $\mathrm{C}$ & -2.7494720 & -3.0736650 & -0.5265320 \\
\hline $\mathrm{C}$ & -1.3891380 & -2.5485470 & -0.8248050 \\
\hline $\mathrm{C}$ & -0.2982980 & -2.8414420 & 0.0049730 \\
\hline $\mathrm{H}$ & -0.4346280 & -3.4813200 & 0.8695720 \\
\hline $\mathrm{C}$ & 0.9674260 & -2.3340040 & -0.2726640 \\
\hline $\mathrm{H}$ & 1.7889310 & -2.5863480 & 0.3857200 \\
\hline $\mathrm{C}$ & 1.1824000 & -1.5047760 & -1.3764740 \\
\hline $\mathrm{C}$ & 2.5490730 & -0.9338000 & -1.7031360 \\
\hline $\mathrm{H}$ & 3.2190430 & -1.7413160 & -1.9968000 \\
\hline $\mathrm{H}$ & 2.4549810 & -0.2678870 & -2.5649780 \\
\hline $\mathrm{C}$ & 2.6081330 & 0.9456220 & -0.1553390 \\
\hline $\mathrm{C}$ & 3.2836370 & 1.7736280 & 0.9310810 \\
\hline $\mathrm{H}$ & 4.1918410 & 2.2133610 & 0.5057590 \\
\hline $\mathrm{H}$ & 3.6114340 & 1.1140780 & 1.7406910 \\
\hline $\mathrm{C}$ & 2.3804600 & 2.8730500 & 1.4978550 \\
\hline $\mathrm{H}$ & 1.4672190 & 2.4150610 & 1.8885470 \\
\hline $\mathrm{H}$ & 2.0688730 & 3.5414280 & 0.6892820 \\
\hline $\mathrm{C}$ & 3.0473130 & 3.6938650 & 2.6127750 \\
\hline $\mathrm{H}$ & 2.2871090 & 4.3480070 & 3.0530720 \\
\hline $\mathrm{H}$ & 3.3704830 & 3.0214180 & 3.4168500 \\
\hline $\mathrm{C}$ & 4.2321500 & 4.5515620 & 2.1533930 \\
\hline $\mathrm{H}$ & 5.0634830 & 3.9436630 & 1.7852920 \\
\hline $\mathrm{H}$ & 4.6149650 & 5.1600080 & 2.9774370 \\
\hline $\mathrm{H}$ & 3.9347990 & 5.2314900 & 1.3482540 \\
\hline $\mathrm{C}$ & -1.1608830 & -1.7609450 & -1.9602180 \\
\hline $\mathrm{H}$ & -1.9861060 & -1.5184270 & -2.6202740 \\
\hline $\mathrm{C}$ & 0.1031820 & -1.2429150 & -2.2242340 \\
\hline
\end{tabular}




\begin{tabular}{|c|c|c|c|}
\hline $\mathrm{H}$ & 0.2446720 & -0.6023170 & -3.0881580 \\
\hline $\mathrm{C}$ & -4.6199260 & -3.4936770 & 0.9797630 \\
\hline $\mathrm{H}$ & -5.0765950 & -3.3631710 & 1.9533630 \\
\hline $\mathrm{C}$ & -5.2847480 & -4.2022600 & -0.0146710 \\
\hline $\mathrm{H}$ & -6.2556280 & -4.6381570 & 0.1887620 \\
\hline $\mathrm{C}$ & -4.6968690 & -4.3376920 & -1.2712970 \\
\hline $\mathrm{H}$ & -5.2052020 & -4.8865020 & -2.0557580 \\
\hline $\mathrm{C}$ & -3.4452800 & -3.7810880 & -1.5171790 \\
\hline $\mathrm{H}$ & -2.9788590 & -3.9181340 & -2.4858290 \\
\hline $\mathrm{C}$ & 4.6430890 & -0.5052450 & -0.3278760 \\
\hline $\mathrm{H}$ & 5.0003980 & 0.2297560 & 0.3877100 \\
\hline $\mathrm{C}$ & 5.4232180 & -0.2566670 & -1.6288620 \\
\hline $\mathrm{C}$ & 4.8903480 & -1.9060210 & 0.2757200 \\
\hline $\mathrm{H}$ & 4.4186690 & -2.6517440 & -0.3724920 \\
\hline $\mathrm{C}$ & 6.3933120 & -2.2228630 & 0.3516720 \\
\hline $\mathrm{H}$ & 6.9228480 & -1.4707860 & 0.9461870 \\
\hline $\mathrm{H}$ & 6.8584090 & -2.2734530 & -0.6340480 \\
\hline $\mathrm{H}$ & 6.5420530 & -3.1899000 & 0.8379710 \\
\hline $\mathrm{C}$ & 4.2707580 & -1.9934030 & 1.6783490 \\
\hline $\mathrm{H}$ & 4.3282050 & -3.0180650 & 2.0534870 \\
\hline $\mathrm{H}$ & 3.2232470 & -1.6886350 & 1.6929090 \\
\hline $\mathrm{H}$ & 4.8154020 & -1.3523740 & 2.3790850 \\
\hline $\mathrm{C}$ & -2.2556360 & 2.3233810 & -0.4188990 \\
\hline $\mathrm{N}$ & -0.9958440 & 2.7611490 & -0.5653810 \\
\hline 0 & -2.5320980 & 1.1597430 & -0.0910960 \\
\hline $\mathrm{H}$ & -0.1986940 & 2.1367110 & -0.4422300 \\
\hline $\mathrm{H}$ & -0.8039910 & 3.6760910 & -0.9413380 \\
\hline $\mathrm{C}$ & -3.3621520 & 3.3053420 & -0.6737700 \\
\hline $\mathrm{C}$ & -3.2116440 & 4.6867110 & -0.5107710 \\
\hline $\mathrm{C}$ & -4.6185200 & 2.8261100 & -1.0541880 \\
\hline $\mathrm{H}$ & -2.2690790 & 5.1075690 & -0.1736610 \\
\hline $\mathrm{C}$ & -5.6444270 & 3.7334440 & -1.2817730 \\
\hline $\mathrm{H}$ & -4.7723060 & 1.7604750 & -1.1694060 \\
\hline $\mathrm{H}$ & -6.6284460 & 3.4017330 & -1.5888980 \\
\hline $\mathrm{N}$ & -4.1951670 & 5.5682340 & -0.7167100 \\
\hline $\mathrm{C}$ & -5.3844880 & 5.0914760 & -1.1043270 \\
\hline $\mathrm{H}$ & -6.1645720 & 5.8268840 & -1.2753330 \\
\hline \multicolumn{2}{|c|}{ Total energy } & \multicolumn{2}{|c|}{-1848.99399892} \\
\hline \multicolumn{2}{|c|}{ Number of imaginary vibrational frequencies } & \multicolumn{2}{|c|}{0} \\
\hline
\end{tabular}




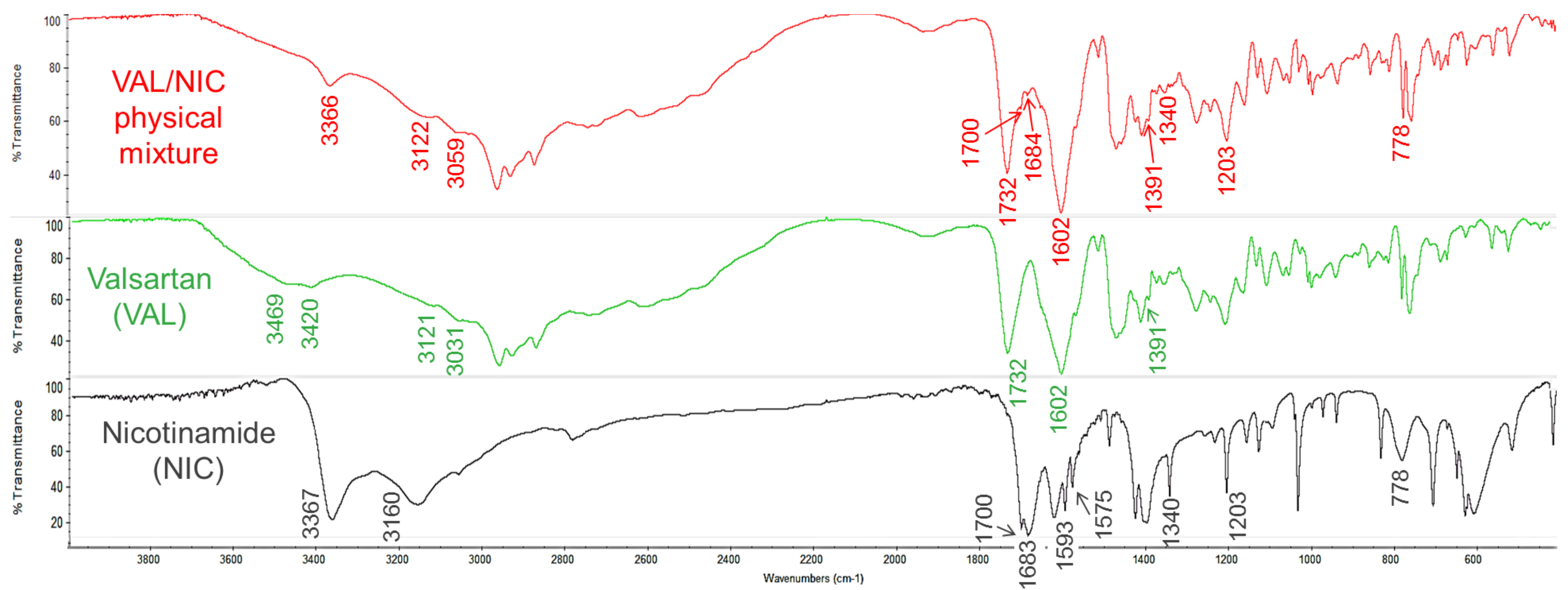

Fig. S11. FT-IR spectra recorded for VAL, NIC and their physical mixture. 


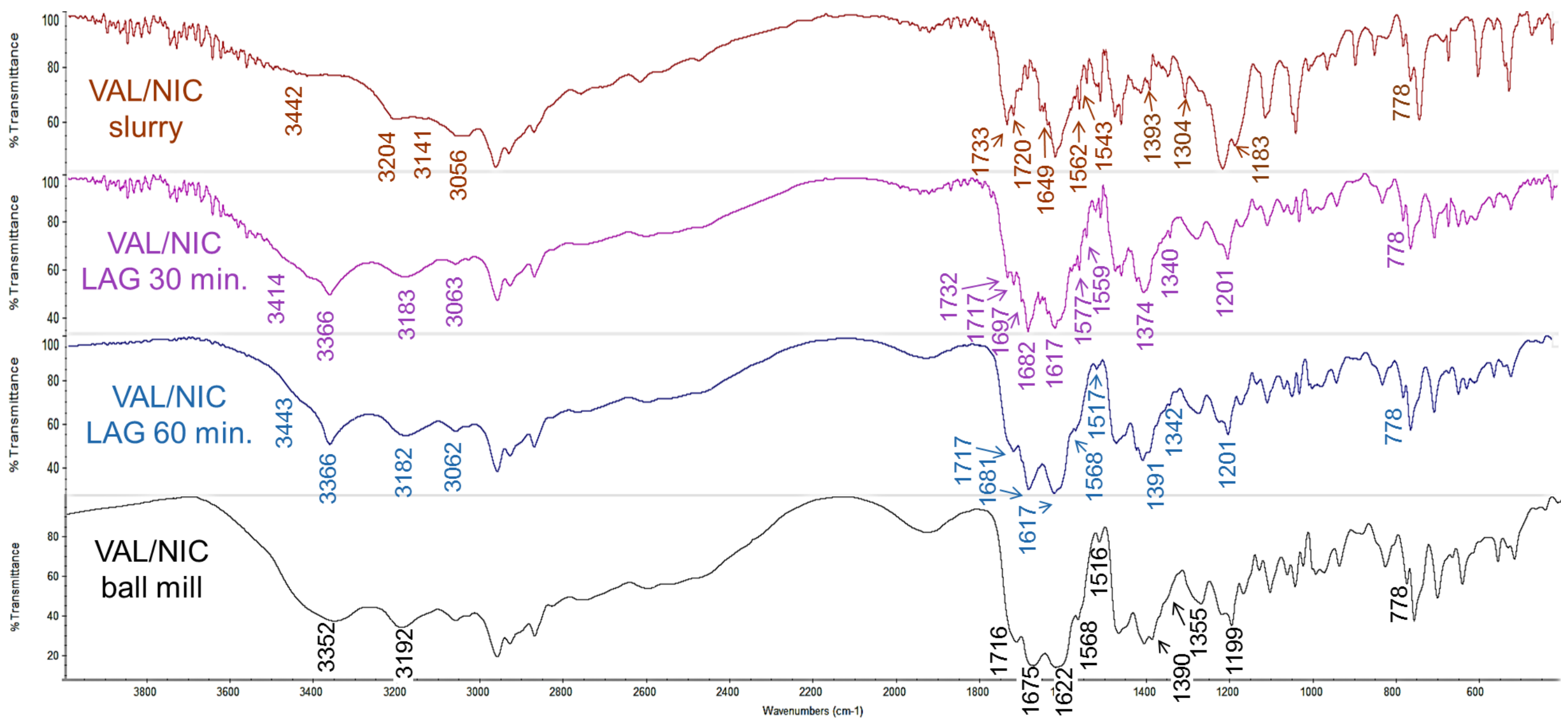

Fig. S12. FT-IR spectra recorded for VAL/NIC co-amorphous formulations. 
Table S6. Comparison of experimental vibrations of VAL and NIC with calculated frequencies along with potential energy distribution (PED, \%). ${ }^{11 *}$

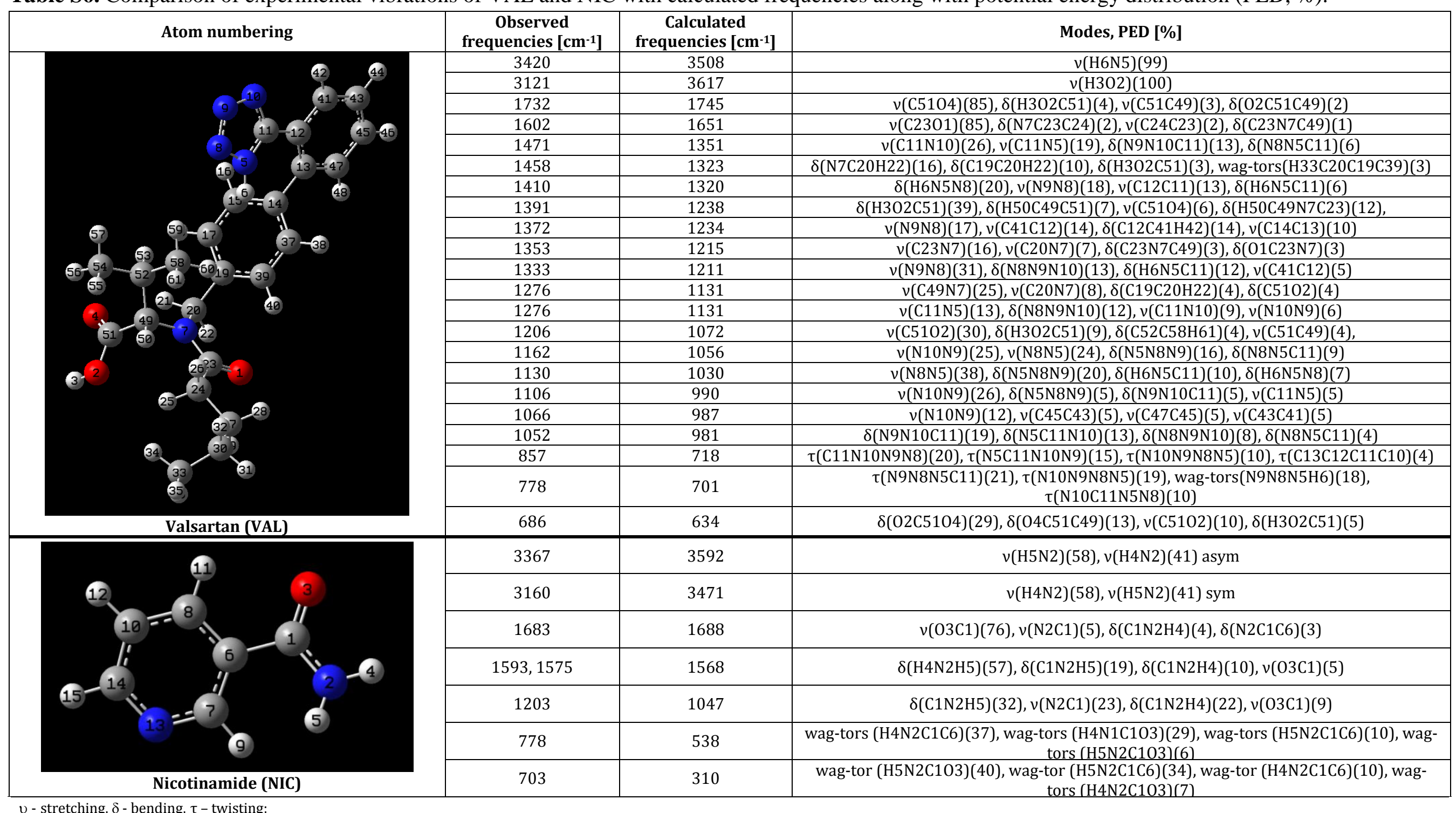

$\mathrm{v}$ - stretching, $\delta$ - bending, $\tau$ - twisting;

*PED matrices were calculated by using the FCART program

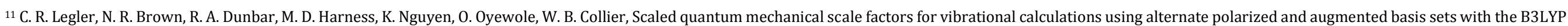
density functional calculation model, Spectrochim. Acta Part A 145 (2015) 15-24 (FCART version 7.0, http://fcart.wbcollier.com/). 
(a)
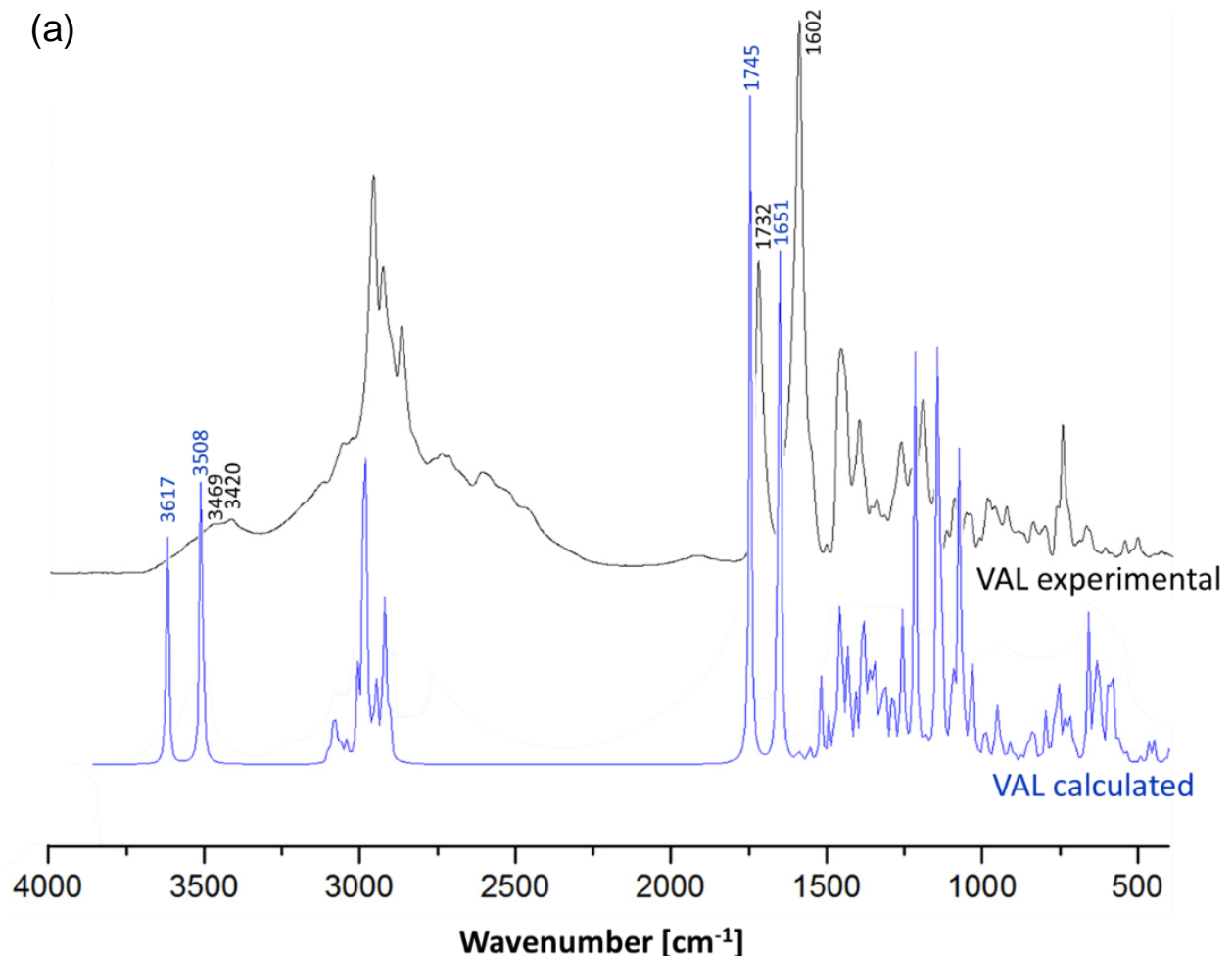

(b)
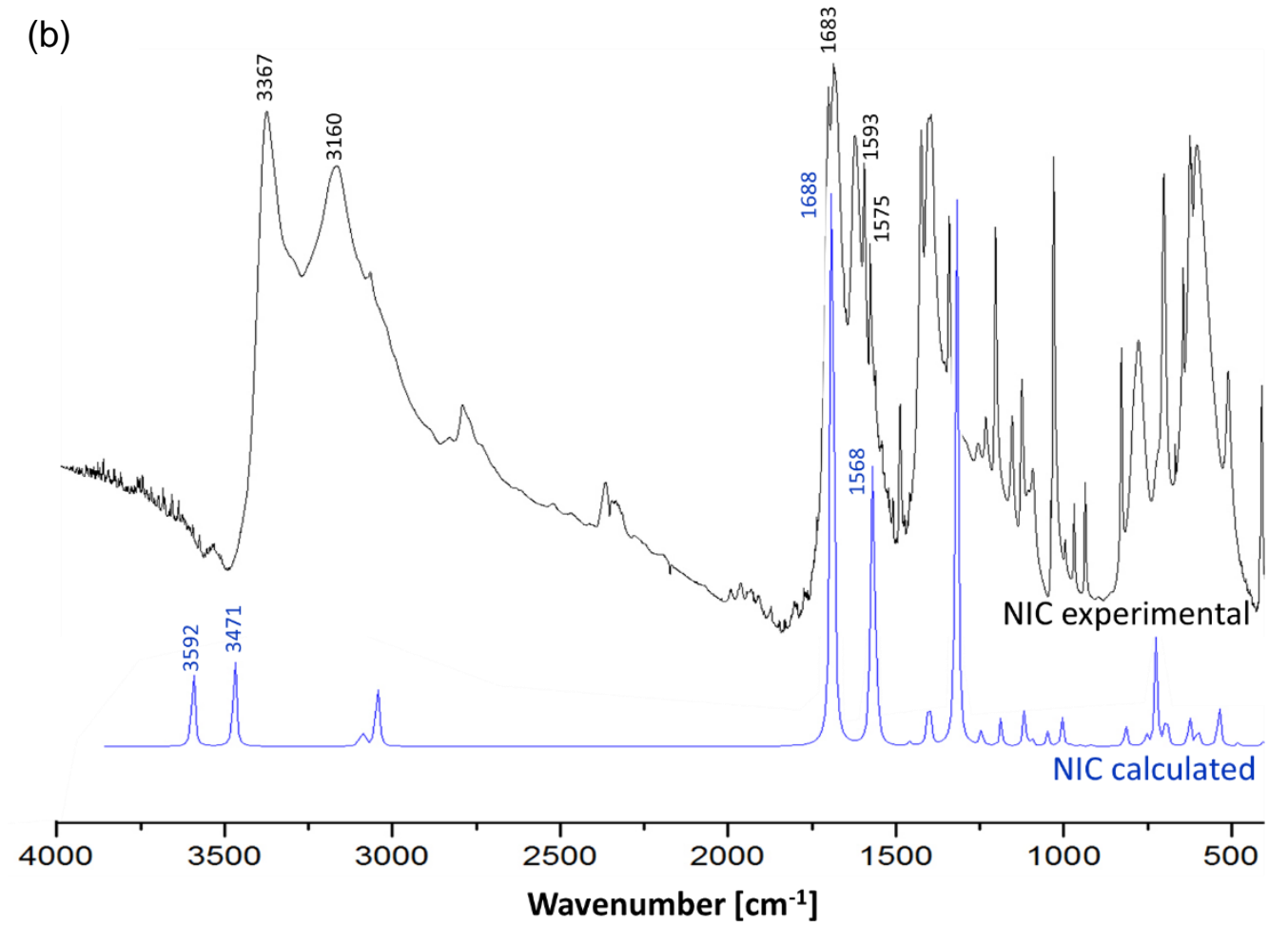

Fig. S13. Comparison of the experimental FT-IR spectrum of VAL (a) and NIC (b) with the theoretical frequencies. 
Table S7. Comparison of experimental vibrations of VAL/NIC slurry with calculated frequencies.

\begin{tabular}{|c|c|c|c|}
\hline \multirow{2}{*}{ Assignment } & \multirow{2}{*}{$\begin{array}{c}\text { Observed frequencies }\left[\mathrm{cm}^{-1}\right] \\
\text { VAL/NIC slurry }\end{array}$} & \multicolumn{2}{|c|}{ Calculated frequencies $\left[\mathrm{cm}^{-1}\right]$} \\
\hline & & VAL/NIC 1 & VAL/NIC 2 \\
\hline$v(\mathrm{OH})$ & 3204 & - & $3619(\Delta=-177)$ \\
\hline$v(\mathrm{NH})^{\text {tetrazole }}$ & 3141 & $3508(\Delta=-367)$ & $3227(\Delta=-86)$ \\
\hline$v(C=0)^{\text {acid }}$ & 1733 & $1686(\Delta=+47)$ & $1751(\Delta=-18)$ \\
\hline$v(C=0)^{\text {amide }}$ & 1649 & $1647(\Delta=+2)$ & $1624(\Delta=+25)$ \\
\hline$v(C-0)$ & 1393 & $1204(\Delta=+189)$ & $1073(\Delta=+320)$ \\
\hline $\mathrm{v}\left(\mathrm{NH}_{2}\right)^{\text {as }}$ & 3204 & $3565(\Delta=-361)$ & $3564(\Delta=-360)$ \\
\hline $\mathrm{v}\left(\mathrm{NH}_{2}\right)^{\mathrm{s}}$ & 3056 & $3278(\Delta=-222)$ & $3340(\Delta=-284)$ \\
\hline$v(C=0)$ & 1720 & $1646(\Delta=+74)$ & $1663(\Delta=+57)$ \\
\hline$\delta(\mathrm{NH})$ & 1562 & $1569(\Delta=-7)$ & $1596(\Delta=-34)$ \\
\hline$\delta(\mathrm{NH})$ & 1543 & $1546(\Delta=-3)$ & $1596(\Delta=-53)$ \\
\hline$v(C-N)$ & 1304 & $1248(\Delta=+56)$ & $1247(\Delta=+57)$ \\
\hline$\rho_{\mathrm{w}}\left(\mathrm{NH}_{2}\right)$ & 778 & $769(\Delta=+9)$ & $735(\Delta=+43)$ \\
\hline
\end{tabular}

Table S8. Comparison of experimental vibrations of VAL/NIC LAG 60 min. with calculated frequencies.

\begin{tabular}{|c|c|c|c|}
\hline \multirow{2}{*}{ Assignment } & \multirow{2}{*}{$\begin{array}{c}\text { Observed frequencies [cm-1] } \\
\text { VAL/NIC LAG } 60 \mathrm{~min} .\end{array}$} & \multicolumn{2}{|c|}{ Calculated frequencies $\left[\mathrm{cm}^{-1}\right]$} \\
\hline & & VAL/NIC 1 & VAL/NIC 2 \\
\hline$v(\mathrm{OH})$ & - & - & $3619(-)$ \\
\hline $\mathrm{v}(\mathrm{NH})^{\text {tetrazole }}$ & 3443 & $3508(\Delta=-65)$ & $3227(\Delta=+216)$ \\
\hline$v(C=0)^{\text {acid }}$ & 1717 & $1686(\Delta=+31)$ & $1751(\Delta=-34)$ \\
\hline$v(C=0)^{\text {amide }}$ & 1605 & $1647(\Delta=-42)$ & $1624(\Delta=-19)$ \\
\hline$v(C-O)$ & 1391 & $1204(\Delta=+187)$ & $1073(\Delta=+318)$ \\
\hline$v\left(\mathrm{NH}_{2}\right)^{\text {as }}$ & 3366 & $3565(\Delta=-199)$ & $3564(\Delta=-198)$ \\
\hline $\mathrm{v}\left(\mathrm{NH}_{2}\right)^{\mathrm{s}}$ & 3182 & $3278(\Delta=-96)$ & $3340(\Delta=-158)$ \\
\hline$v(C=0)$ & 1681 & $1646(\Delta=+35)$ & $1663(\Delta=+18)$ \\
\hline$\delta(\mathrm{NH})$ & 1568 & $1569(\Delta=-1)$ & $1596(\Delta=-28)$ \\
\hline$\delta(\mathrm{NH})$ & 1517 & $1546(\Delta=-29)$ & $1596(\Delta=-79)$ \\
\hline$v(C-N)$ & 1342 & $1248(\Delta=+94)$ & $1247(\Delta=+95)$ \\
\hline$\rho_{\mathrm{w}}\left(\mathrm{NH}_{2}\right)$ & 778 & $769(\Delta=+9)$ & $735(\Delta=+43)$ \\
\hline
\end{tabular}

Table S9. Comparison of experimental vibrations of VAL/NIC ball mill with calculated frequencies.

\begin{tabular}{|c|c|c|c|}
\hline \multirow{2}{*}{ Assignment } & \multirow{2}{*}{$\begin{array}{c}\text { Observed frequencies [cm-1] }^{-1} \\
\text { VAL/NIC ball mill }\end{array}$} & \multicolumn{2}{|c|}{ Calculated frequencies $\left[\mathrm{cm}^{-1}\right]$} \\
\hline & & VAL/NIC 1 & VAL/NIC 2 \\
\hline$v(\mathrm{OH})$ & - & - & $3619(-)$ \\
\hline$v(\mathrm{NH})^{\text {tetrazole }}$ & 3352 & $3508(\Delta=-156)$ & $3227(\Delta=+125)$ \\
\hline$v(C=0)^{\text {acid }}$ & 1716 & $1686(\Delta=+30)$ & $1751(\Delta=-35)$ \\
\hline$v(C=0)^{\text {amide }}$ & 1622 & $1647(\Delta=-25)$ & $1624(\Delta=-2)$ \\
\hline$v(C-0)$ & 1390 & $1204(\Delta=+186)$ & $1073(\Delta=+317)$ \\
\hline$v\left(\mathrm{NH}_{2}\right)^{\text {as }}$ & 3352 & $3565(\Delta=-213)$ & $3564(\Delta=-212)$ \\
\hline $\mathrm{v}\left(\mathrm{NH}_{2}\right)^{\mathrm{s}}$ & 3192 & $3278(\Delta=-86)$ & $3340(\Delta=-148)$ \\
\hline$v(C=0)$ & 1675 & $1646(\Delta=+29)$ & $1663(\Delta=+12)$ \\
\hline$\delta(\mathrm{NH})$ & 1568 & $1569(\Delta=-1)$ & $1596(\Delta=-28)$ \\
\hline$\delta(\mathrm{NH})$ & 1516 & $1546(\Delta=-30)$ & $1596(\Delta=-80)$ \\
\hline$v(C-N)$ & 1355 & $1248(\Delta=+107)$ & $1247(\Delta=+108)$ \\
\hline$\rho_{\mathrm{w}}\left(\mathrm{NH}_{2}\right)$ & 778 & $769(\Delta=+9)$ & $735(\Delta=+43)$ \\
\hline
\end{tabular}




\subsection{Quantum Theory of Atoms in Molecules (QTAIM)}

Table S10. Topological parameters corresponding to H-bonds involved in intermolecular interactions.

\begin{tabular}{|c|c|c|c|c|c|c|c|c|c|}
\hline D-H $\cdots A$ & $\rho_{B C P}$ & $\nabla^{2} \rho_{B C P}$ & $G_{B C P}$ & $V_{B C P}$ & $\boldsymbol{H}_{B C P}$ & $K_{B C P}$ & $\mathrm{E}_{b i n}[\mathrm{kcal} / \mathrm{mol}]$ & $\begin{array}{c}\Delta \mathbf{r}_{\mathrm{H}}+ \\
\Delta \mathbf{r}_{\mathrm{A}}\end{array}$ & $\begin{array}{c}\Delta \mathbf{r}_{\mathrm{H}}- \\
\Delta \mathbf{r}_{\mathrm{A}}\end{array}$ \\
\hline \multicolumn{10}{|c|}{ Calculated in gas phase } \\
\hline \multicolumn{10}{|c|}{ VAL/NIC1 } \\
\hline $\mathrm{O} 2-\mathrm{H} 2 \cdots 04$ & 0.0514 & 0.1413 & 0.0427 & -0.0500 & -0.0073 & 0.0073 & -15.68 & 1.08 & 0.28 \\
\hline N3-H3a $\cdots 03$ & 0.0271 & 0.0967 & 0.0223 & -0.0204 & 0.0019 & -0.0019 & -6.40 & 0.81 & 0.25 \\
\hline \multicolumn{10}{|c|}{ VAL/NIC2 } \\
\hline $\mathrm{N} 1-\mathrm{H} 1 \cdots \mathrm{O} 4$ & 0.0316 & 0.1229 & 0.0290 & -0.0273 & 0.0017 & -0.0017 & -8.56 & 0.93 & 0.23 \\
\hline N3-H3a $\cdots 01$ & 0.0231 & 0.0938 & 0.0204 & -0.0174 & 0.0030 & -0.0030 & -5.45 & 0.78 & 0.22 \\
\hline \multicolumn{10}{|c|}{ VAL/NIC1 + EtOH } \\
\hline $\mathrm{O} 2-\mathrm{H} 2 \cdots 04$ & 0.0507 & 0.1408 & 0.0421 & -0.0490 & -0.0069 & 0.0069 & -15.36 & 1.08 & 0.27 \\
\hline N3-H3a $\cdots 03$ & 0.0274 & 0.0976 & 0.0226 & -0.0207 & 0.0018 & -0.0018 & -6.49 & 0.81 & 0.25 \\
\hline 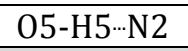 & 0.0195 & 0.0656 & 0.0141 & -0.0119 & 0.0023 & -0.0023 & -3.72 & 0.60 & 0.30 \\
\hline \multicolumn{10}{|c|}{ Calculated in ethanol } \\
\hline \multicolumn{10}{|c|}{ VAL/NIC1 } \\
\hline $\mathrm{O} 2-\mathrm{H} 2 \cdots 04$ & 0.0535 & 0.1436 & 0.0443 & -0.0528 & -0.0084 & 0.0084 & -16.56 & 1.09 & 0.27 \\
\hline N3-H3a $\cdots 03$ & 0.0241 & 0.0858 & 0.0194 & -0.0173 & 0.0021 & -0.0021 & -5.42 & 0.75 & 0.25 \\
\hline $\mathrm{N} 1-\mathrm{H} 1 \cdots \mathrm{O} 4$ & 0.0360 & 0.1333 & 0.0330 & -0.0326 & 0.0003 & -0.0003 & -10.22 & 0.97 & 0.23 \\
\hline N3-H3a $\cdots 01$ & 0.0233 & 0.0948 & 0.0207 & -0.0177 & 0.0030 & -0.0030 & -5.55 & 0.79 & 0.23 \\
\hline
\end{tabular}


Table S11. Geometrical parameters corresponding to H-bonds involved in intermolecular interactions along with molecular graphs with the BCPs (3, -1) as orange dots.

\begin{tabular}{|c|c|c|c|c|c|}
\hline Designed system & D-H $\cdots A$ & D-H [Å] & 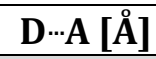 & 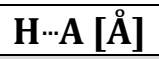 & $\mathrm{D}-\mathrm{H} \cdots \mathrm{A}\left[{ }^{\circ}\right]$ \\
\hline \multicolumn{6}{|c|}{ Calculated in gas phase } \\
\hline \multirow{2}{*}{ VAL/NIC1 } & $\mathrm{O} 2-\mathrm{H} 2 \cdots 04$ & 1.006 & 2.642 & 1.638 & 175.234 \\
\hline & N3-H3a $\cdots 03$ & 1.022 & 2.911 & 1.909 & 165.671 \\
\hline \multirow{2}{*}{ VAL/NIC2 } & $\mathrm{N} 1-\mathrm{H} 1 \cdots 04$ & 1.027 & 2.809 & 1.794 & 168.946 \\
\hline & N3-H3a‥01 & 1.019 & 2.915 & 1.936 & 160.112 \\
\hline \multirow{3}{*}{ VAL/NIC1 + EtOH } & $\mathrm{O} 2-\mathrm{H} 2 \cdots 04$ & 1.006 & 2.646 & 1.642 & 175.332 \\
\hline & $\mathrm{N} 3-\mathrm{H} 3 \mathrm{a} \cdots \mathrm{O} 3$ & 1.022 & 2.906 & 1.904 & 165.816 \\
\hline & O5-H5 - N2 & 0.969 & 2.986 & 2.100 & 151.243 \\
\hline \multicolumn{6}{|c|}{ Calculated in ethanol } \\
\hline \multirow{2}{*}{ VAL/NIC1 } & $\mathrm{O} 2-\mathrm{H} 2 \cdots 04$ & 1.009 & 2.627 & 1.623 & 172.639 \\
\hline & N3-H3a $\cdots 03$ & 1.020 & 2.960 & 1.962 & 165.225 \\
\hline \multirow{2}{*}{ VAL/NIC2 } & $\mathrm{N} 1-\mathrm{H} 1 \cdots 04$ & 1.033 & 2.764 & 1.747 & 167.435 \\
\hline & N3-H3a $\cdots 01$ & 1.020 & 2.921 & 1.930 & 163.045 \\
\hline \multicolumn{3}{|c|}{ VAL/NIC1 } & \multicolumn{3}{|c|}{ VAL/NIC1 + EtOH } \\
\hline
\end{tabular}



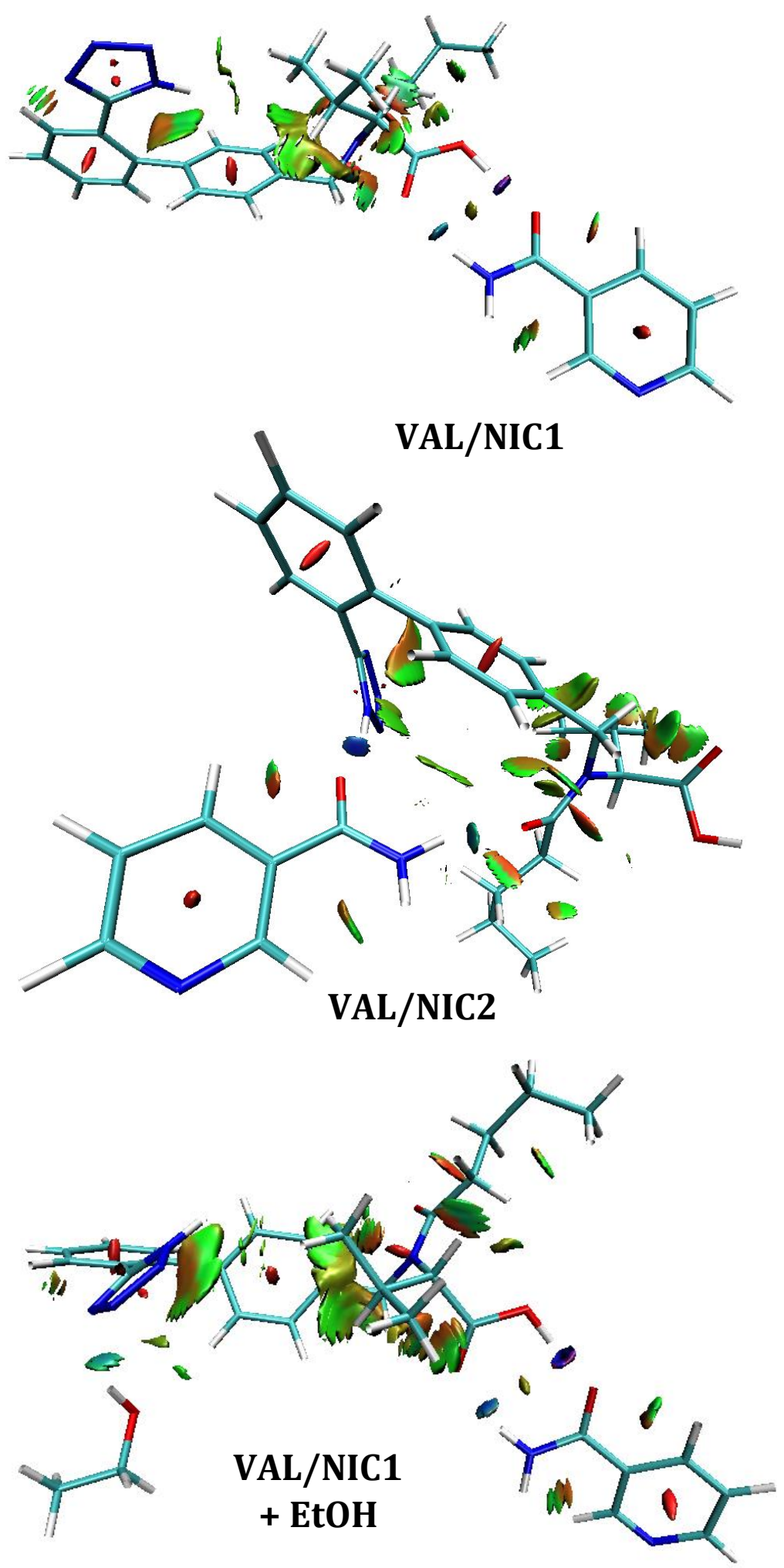
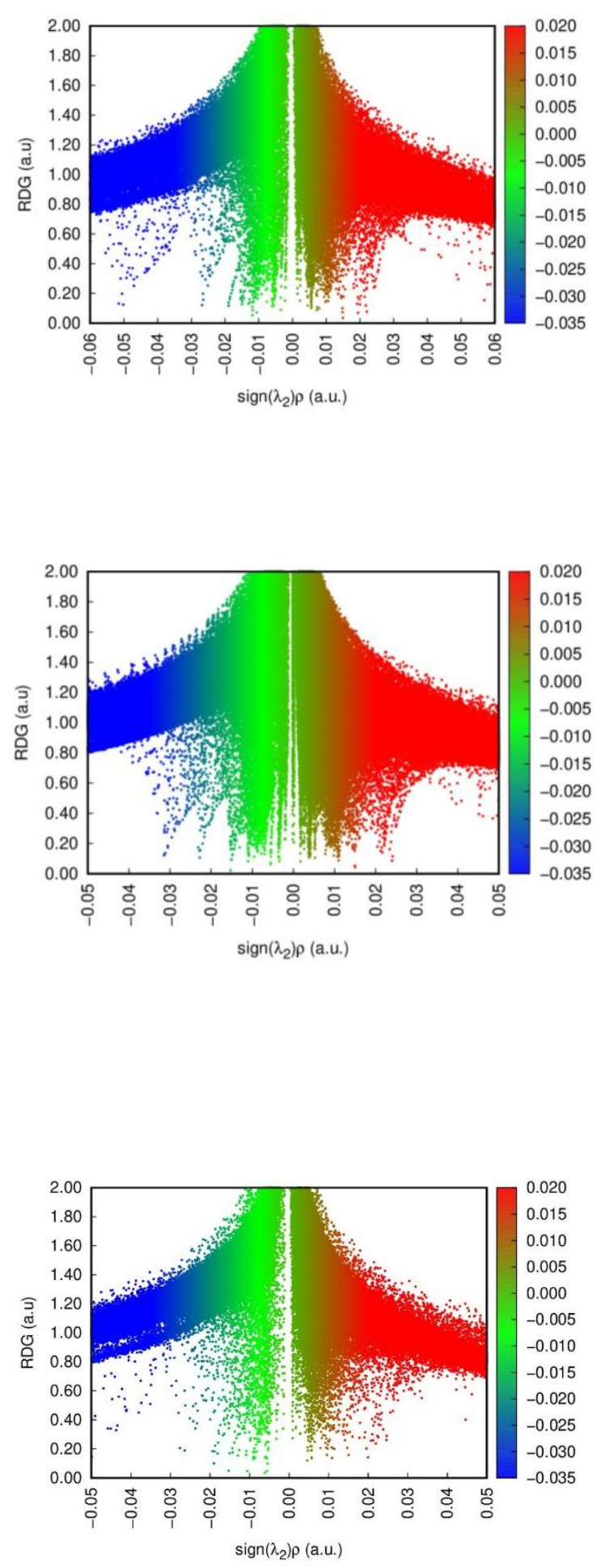

Fig. S14a. 3D-NCI plot with color-filled reduced density gradient (RDG) isosurfaces depicting noncovalent interactions in VAL/NIC1 and VAL/NIC2 along with 2D-NCI scatter plots (calculated in gas phase). 


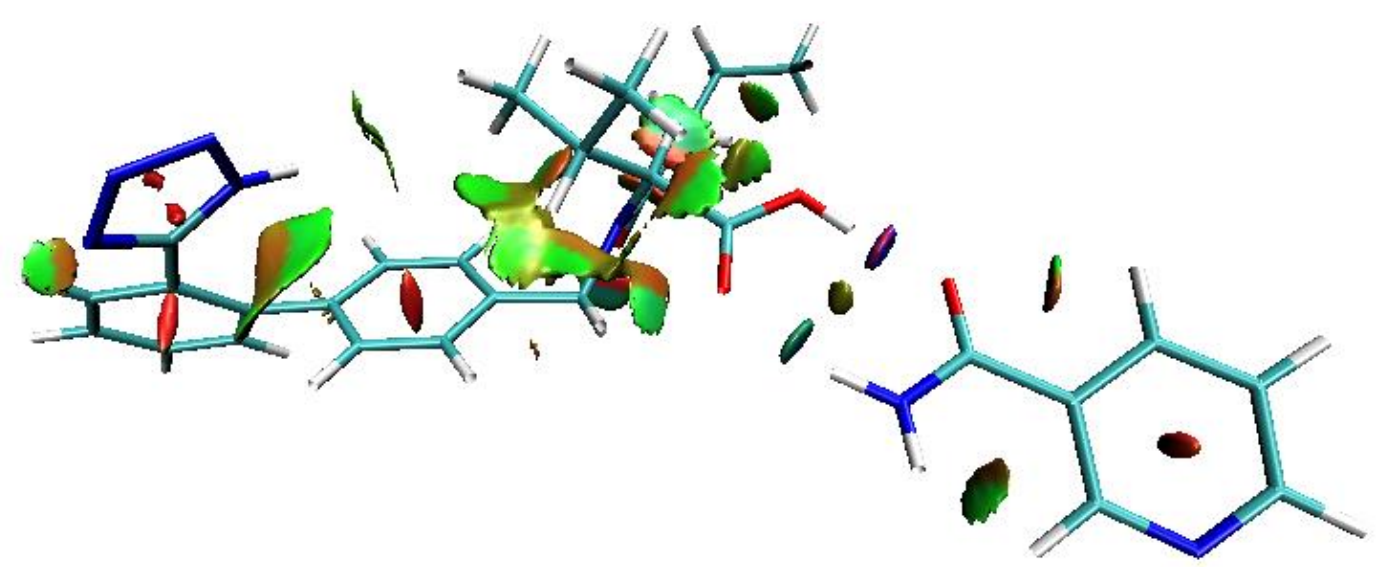

VAL/NIC 1

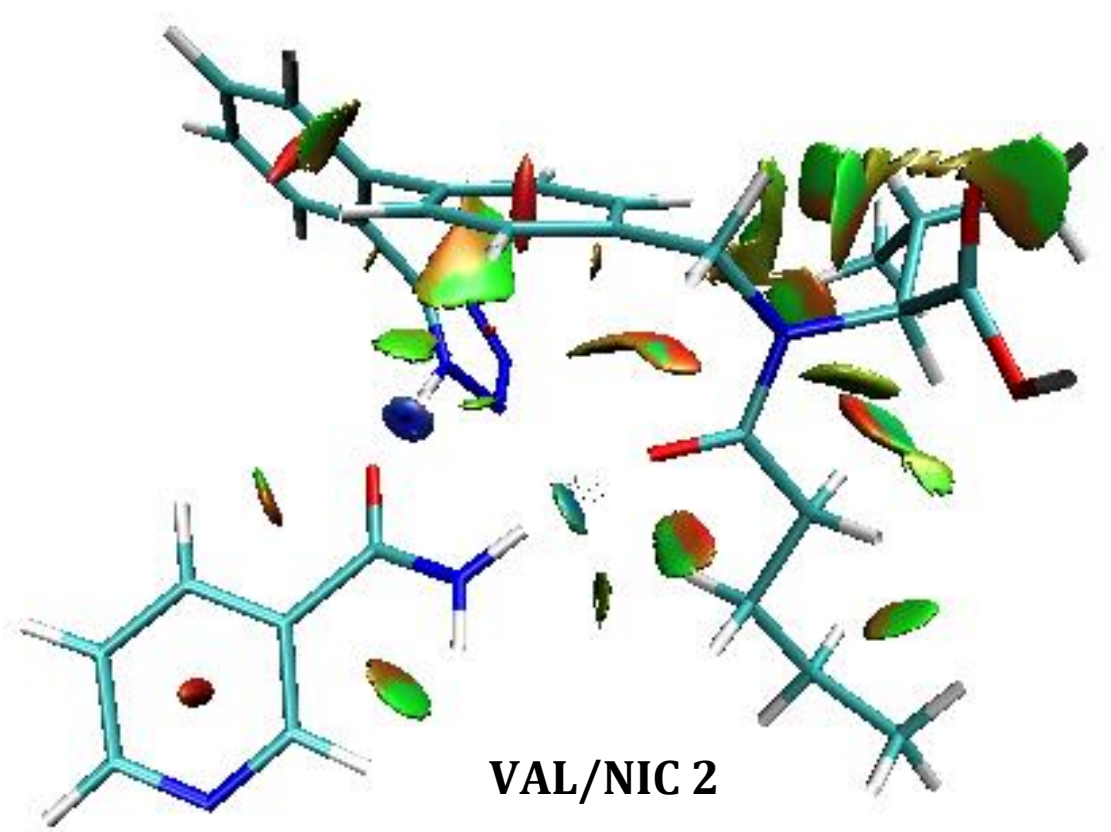

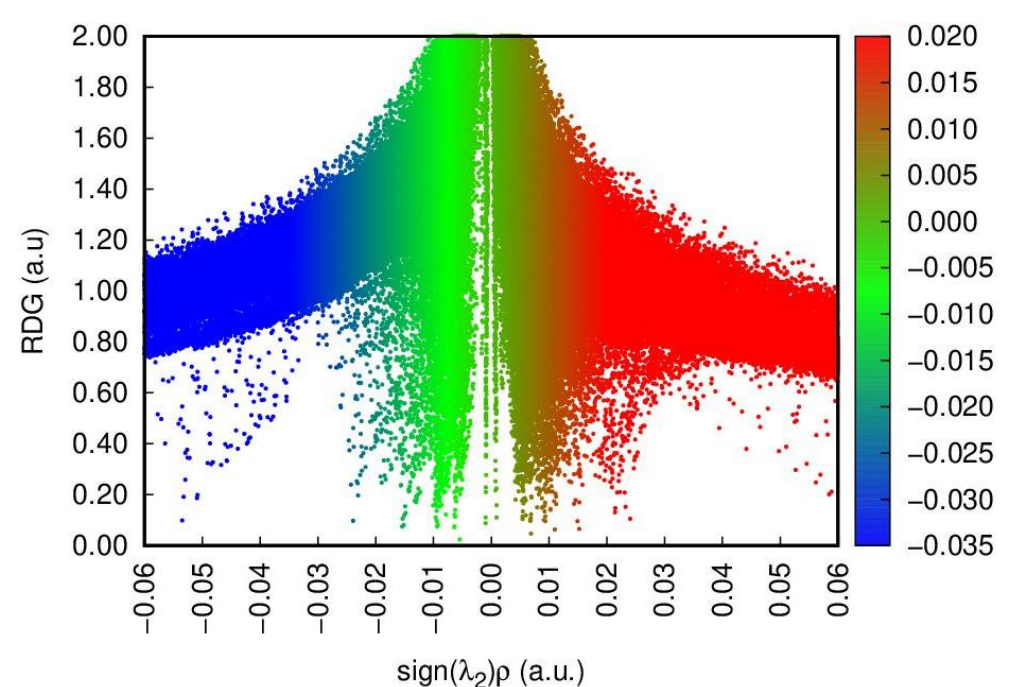

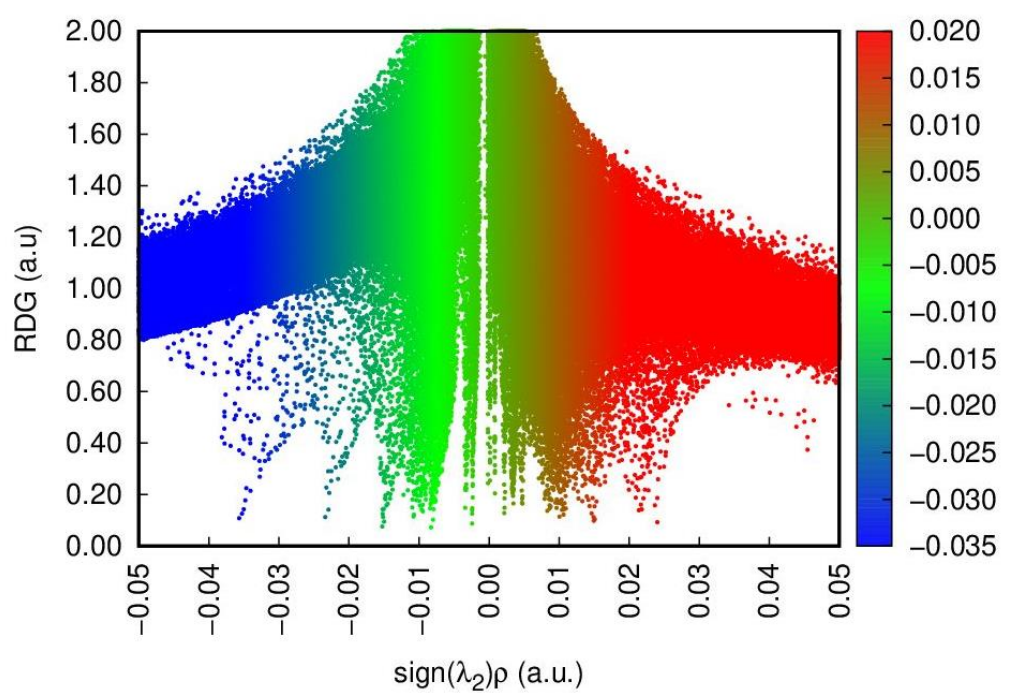

Fig. S14b. 3D-NCI plot with color-filled reduced density gradient (RDG) isosurfaces depicting non-covalent interactions in VAL/NIC1 and VAL/NIC2 along with 2D-NCI scatter plots (calculated in ethanol). 


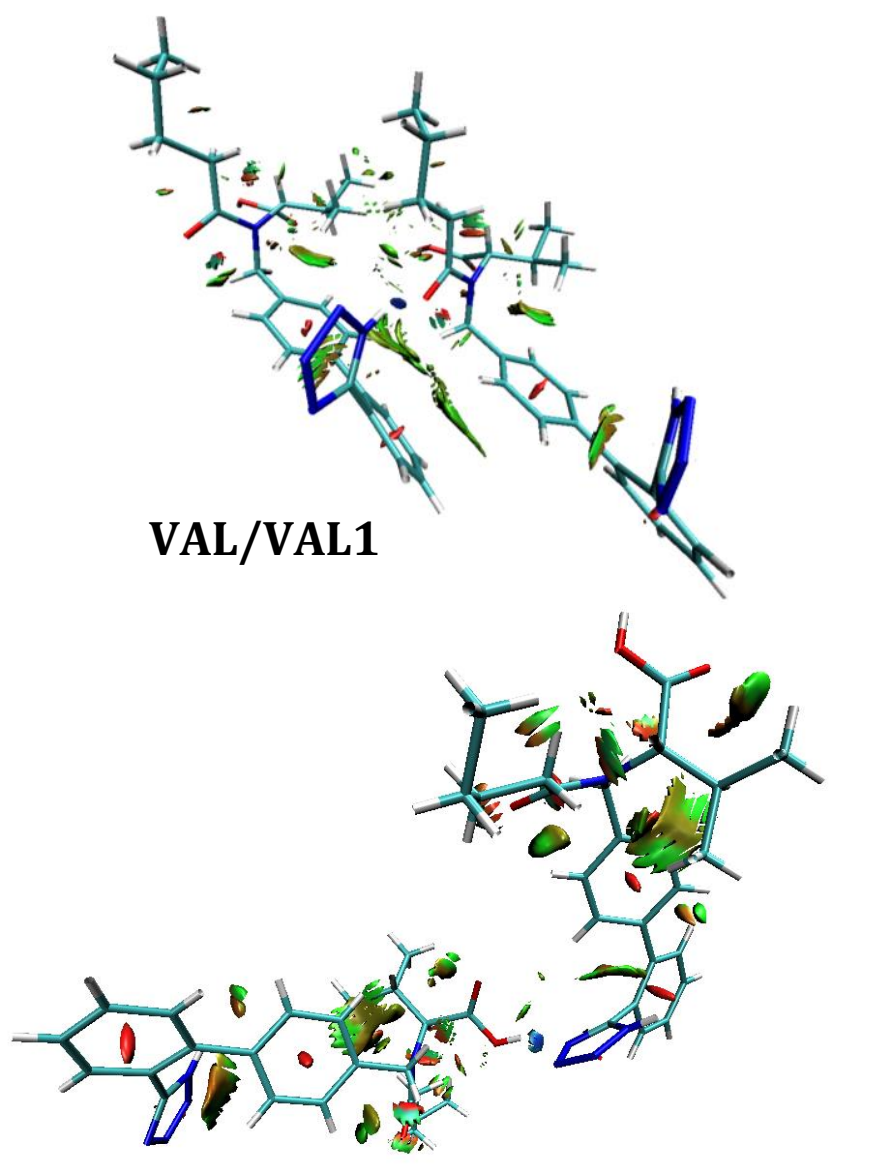

VAL/VAL2

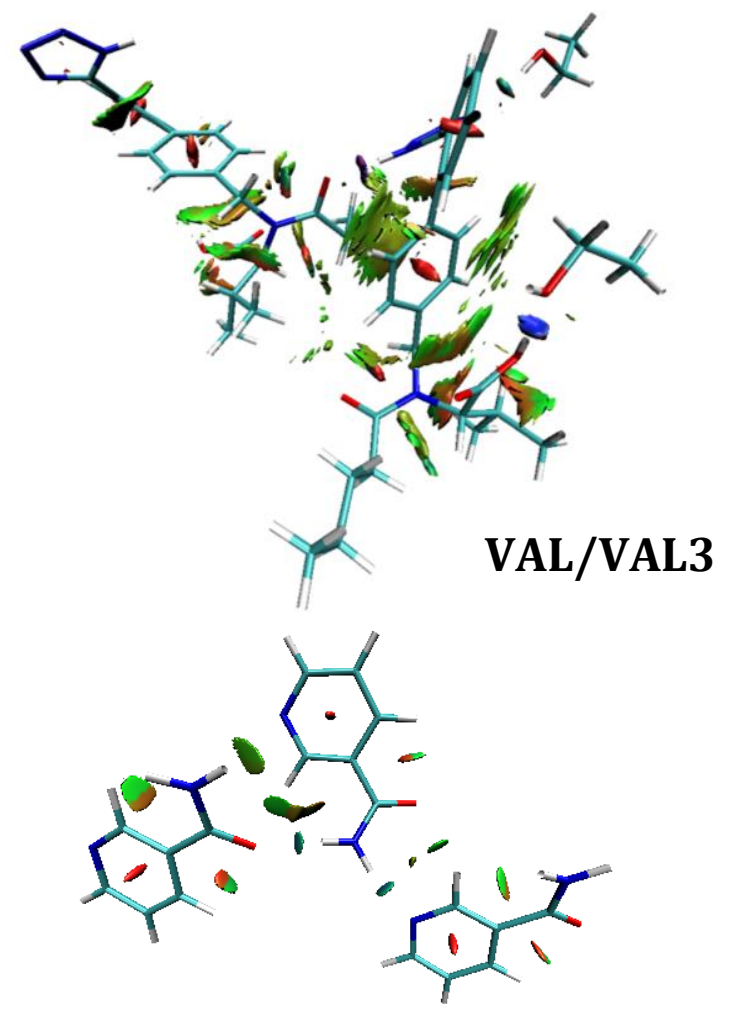

NIC/NIC
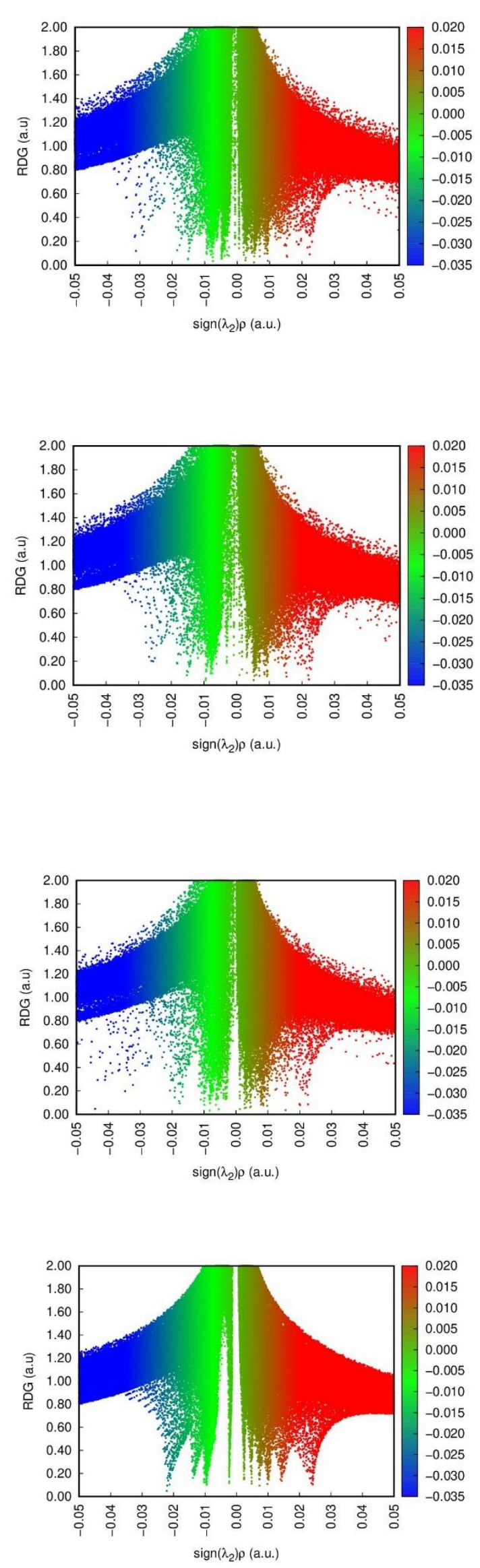

Fig. S15. 3D-NCI plot with color-filled reduced density gradient (RDG) isosurfaces depicting noncovalent interactions in homodimers/trimers along with 2D-NCI scatter plots. 